Col I i si onal i ty dependence and i on speci es effects on heat transport in He and H pl asma, and the role of $i$ on scal e turbul ence in LHD

\begin{tabular}{|l|l|}
\hline $\begin{array}{l}\text { j our nal or } \\
\text { publ i cat } \mathrm{i} \text { on } \mathrm{t} \text { i t l e }\end{array}$ & Nucl ear Fusi on \\
\hline vol une & 57 \\
\hline number & 11 \\
\hline page $\mathrm{r}$ ange & 116005 \\
\hline year & $2017-08$ 01 \\
\hline URL & ht t p: //hdl . handl e. net /10655/00012633 \\
\hline
\end{tabular}




\title{
Collisionality dependence and ion species effects on heat transport in $H e$ and $H$ plasma and the role of ion scale turbulence in LHD
}

\author{
K. Tanaka ${ }^{1,2}$, K. Nagaoka ${ }^{1}$, S. Murakami ${ }^{3}$, H. Takahashi ${ }^{1,4}$, M. Osakabe ${ }^{1,4}$, M. Yokoyama ${ }^{1,4}$, R. \\ Seki $^{1,4}$, C. A. Michael ${ }^{5}$, H. Yamaguchi ${ }^{3}$, C. Suzuki ${ }^{1}$, A. Shimizu ${ }^{1}$, T. Tokuzawa ${ }^{1}$, M. Yoshinuma ${ }^{1,4}$, T. \\ Akiyama $^{1}$, K. Ida ${ }^{1,4}$, I. Yamada ${ }^{1}$, R. Yasuhara ${ }^{1}$, H. Funaba ${ }^{1}$, T. Kobayashi ${ }^{1,4}$, H. Yamada ${ }^{1}$, X.D. Du ${ }^{1}$, L. \\ N. Vyacheslavov ${ }^{6}$, D. R. Mikkelsen ${ }^{7}$, G. S. Yun ${ }^{8}$ and the LHD Experimental Group ${ }^{1}$
}

${ }^{1}$ National Institute for Fusion Science, Toki 509-5292, Japan

${ }^{2}$ Kyushu University, Department of Advanced Energy Engineering, Kasuga, Fukuoka, 816-8580, Japan

${ }^{3}$ Department of Nuclear Engineering, Kyoto University, Kyoto 615-8540, Japan

${ }^{4}$ SOKENDAI (The Graduate University for Advanced Studies), Toki, Gifu, 509-5292, Japan

${ }^{5}$ Plasma Research Lab, Australian National University, Canberra, A.C.T. 2601, Australia

${ }^{6}$ Budker Institute of Nuclear Physics, 630090 Novosibirsk, Russian Federation

${ }^{7}$ Princeton Plasma Physics Laboratory, Princeton, New Jersey 08543, USA

${ }^{8}$ Pohang University of Science and Technology, Pohang, Gyeongbuk 37673, Korea

\section{E-mail contact of main author: ktanaka@nifs.ac.jp}

\begin{abstract}
The surveys of the ion and electron heat transports of Neutral Beam (NB) heating plasma were carried out by power balance analysis in He and H rich plasma of LHD. Collisionality was scanned by changing density and heating power. The characteristics of the transport vary depending on collisionality. In low collisionality with low density and high heating power, ion internal transport barrier (ITB) was formed. The ion heat conductivities $\left(\chi_{i}\right)$ is lower than electron heat conductivities $\left(\chi_{e}\right)$ in the core region at $\rho<0.7$. On the other hand, in high collisionality with high density and low heating power, $\chi_{i}$ is higher than $\chi_{\mathrm{e}}$ in the entire region of the plasma. These different confinement regimes are associated with different fluctuation characteristics. In ion ITB, fluctuation has a peak at $\rho=0.7$, and in normal confinement, fluctuation has a peak at $\rho=1.0$. The two confinement mode changes gradually depending on the collisionality. The scan of concentration ratio between $\mathrm{He}$ and $\mathrm{H}$ were also performed. The ion confinement improvements were investigated by gyro-Bohm normalization taking account of the effective mass and charge. The concentration ratio affected the normalized $\chi_{i}$ only in the edge region $(\rho \sim 1.0)$. This indicates ion species effects vary depending on collisionality. Turbulence was modulated by the fast ion loss instability. The modulation of turbulence is higher in $\mathrm{H}$ rich than in He rich plasma.
\end{abstract}




\section{Introduction}

LHD is the largest helical device presently working as well as W7-X. LHD is categorized as a heliotron [1]. Heliotron has been developed since 1950s in Japan. Heliotron is characterized by the combination of the helical winding coils and the vertical coils to produce confinement magnetic field. Helical pitch is 10 indicating that the elliptical shape of plasma is rotating 10 times around the torus. Major radius is $3.5-4 \mathrm{~m}$, minor radius is $0.6 \mathrm{~m}$. The plasma currentless operation is possible. This is the important advantage for steady state operation. It is a significant contrast to tokamak that no necessity of plasma current control to keep confinement magnetic field. The control of coil current enables changing magnetic properties such as iota profile, iota shear, helical ripple and magnetic field curvature. The change of the magnetic properties results in the change of MHD stability and the transport, which are both neoclassical and turbulence driven-anomalous transports.

The pumped closed helical divertor was installed in the torus inboard side at the 6 of the 10 toroidal sections in 2014[2]. The maximum injection power of ECRH is 5.4MW (three $1-1.5 \mathrm{MW} 77 \mathrm{GHz}$ and two $1 \mathrm{MW} 154 \mathrm{GHz}$ gyrotrons). The maximum injection power of NBI is 28MW (6MW, 5MW and 5MW negative ion based tangential injections, and two $6 \mathrm{MW}$ positive ion based perpendicular injections). Also, 4MW ICRH are available. Excellent profile diagnostics are also available. YAG laser Thomson scattering measures electron density and temperature with fine resolution. Charge exchange spectroscopy measures ion temperature, plasma rotation and carbon impurity profiles. Far infrared laser and $\mathrm{CO}_{2}$ laser interferometer measures continuous electron density evolution and provides radial density profiles by Abel inversion. Such fine profile diagnostics are great advantages for transport study. Details regarding machine specifications including heating and diagnostics and physics results are reviewed in ref.3. Also, reactor design study based on LHD is in progress [4]

In helical devices, scaling study of global energy confinement were performed for small and medium size devices $[5,6,7]$ and extended using LHD data $[8,9]$. The latest scaling is called international stellarator scaling 2004 (ISS04)[9]. ISS04 follows gyro-Bohm scaling and is dimensionally equivalent to tokamak ELMy H mode scaling (ITER98y2 [10]) [9]. There are documentations of transport studies in LHD. Heat transport of high beta regimes were reported in ref.11 and 12. Confinement degradation of edge $(\rho=0.9)$ heat conductivity at volume averaged beta higher than $1 \%$ was reported $[11,12]$. Particle transport of LHD is reviewed in ref.13. Increase of hollowness with decrease of collisionality and shifting magnetic axis outwardly is consistent with increase of 
neoclassical thermos-diffusion, while diffusion coefficients are much higher than neoclassical coefficients. Impurity transports are reviewed in ref.14. Accumulation of impurity was summarized in density - temperature diagram. It was emphasized that the effect of impurity source location and impurity transport should be distinguished.

Inter-machine comparison of helical devices are also performed. Core electron-root confinement (CERC) were achieved in W7-AS, TJ-II, CHS and LHD. The improvements of core electron heat transport are well understood to be due to the transition of electron root associated with large positive radial electric field [15]. Comparative study of electron and ion transports in mid to high density regime of W7-AS, T-JII, and LHD were reported in ref.16. The radial electric field agrees with neoclassical prediction. Electron and ion transport were governed by neoclassical prediction in W7-AS but not in other devises, while edge (outer of $2 / 3$ minor radius) heat transport is governed by anomalous transport in all devices.

There are reports of transport comparison between hydrogen isotopes (hydrogen and deuterium). In W7-AS, an increase of approximately $20 \%$ of energy confinement in the ECRH deuterium plasma than in the hydrogen plasma were reported [17]. In CHS, lower particle diffusivity in the NBI heating deuterium plasma than in the hydrogen are reported in low density regime, while energy transport improvements were not found [18]. In Heliotron-J, lower particle diffusivity in the ECRH deuterium plasma than in hydrogen were reported [19]. In TJ-II, degradation of impurity confinement with increase of deuterium contents are reported [20]. In helical device, hydrogen isotope effects are not common, but vary depending on transport channel and devices. On the other hand, in tokamak, better transport characteristics in deuterium than in hydrogen plasma are widely observed [21-26]. Lower $\mathrm{H}$ mode threshold power and lower ion thermal conductivity in the deuterium plasma than in hydrogen plasma are reported $[25,26]$.

On the other hand, comparison study of transport between helium and hydrogen isotopes are very limited both in tokamak and helical devices. In ASDEX-U, H mode threshold power is comparable in the deuterium and helium and is lower than in the hydrogen. Global energy confinement is the highest in deuterium, followed by helium and is the lowest in the hydrogen plasma [26]. Comparable particle transport in the deuterium and helium plasma was reported in ASDEX, suggesting that the mass number per charge determines the particle transport [27]. In LHD, comparison studies were carried out in ECRH plasma in the hydrogen and helium plasma. Comparable electron heat transport and better ion heat transport in the helium plasma are reported [28].

In this paper, we report the results of the characteristics of ion and electron heat transport from the scan of collisionality and ion concentration ratio between $\mathrm{H}^{+}$and $\mathrm{He}^{2+}$ in LHD 
in order to understand ion species effects on the heat transport. This study is the first step, and will be extended by the result of deuterium experiments of LHD, which begins from 2017. The initial results of performance of He- and H-rich plasma were reported in ref. 29. Detailed transport analysis and turbulence characteristics are described in the following sections for the first time.

\section{Transport analysis of $\mathbf{H e}$ and $H$ rich plasma in normal confinement and ion ITB}

Figure 1 shows the achieved $T_{e}, T_{i}$ in the central region $(\rho<0.3)$ of different ion concentration ratio. The ratio of the $\mathrm{H}^{+}$and $\mathrm{He}^{2+}$ ions were estimated from the ratio of influx, which consists of external fueling and wall recycling. We define the concentration ratio as $\mathrm{R}=\mathrm{n}_{\mathrm{H}} /\left(\mathrm{n}_{\mathrm{H}}+\mathrm{n}_{\mathrm{He}}\right)$, where $\mathrm{n}_{\mathrm{H}}$ is $\mathrm{H}^{+}$ion density and $\mathrm{n}_{\mathrm{He}}$ is $\mathrm{He}^{2+}$ ion density. The concentration ratio was estimated from intensities of the $\mathrm{H \alpha}(656.3 \mathrm{~nm})$ and HeI $(587.6$ $\mathrm{nm}$ ) lines. Each line intensity is proportional to the neutral influx and approximately equal to ion density of the $\mathrm{H}^{+}$and $\mathrm{He}^{2+}$, respectively. Each line intensities were absolutely calibrated and were measured at 10 toroidal sections. The averaged intensities were used to estimate the concentration ratio and the concentration ratio was assumed to be spatially constant in plasma. The experiments were performed at $\mathrm{Bt}=2.75 \mathrm{~T}$ and the magnetic axis position $\left(R_{a x}\right)$ was $3.6 \mathrm{~m}$. $R_{a x}=3.6 \mathrm{~m}$ is the best configuration of performance of low $\beta$ plasma [9].

The results shown in Fig.1 consist of two series of experiments. One is low density high power, which is so-called "ion ITB plasma". The set of ion ITB plasmas in Fig.1 were heated by a $20-23 \mathrm{MW}$ hydrogen neutral beam. Around $11 \mathrm{MW}$ was positive ion-based perpendicular injected NBI (perp-PNB), which predominantly heated ions, and the other 9-12MW was negative ion-based parallel injected NBI (para-NNB), which predominantly heated electrons. The line averaged density was $1.35 \times 10^{19} \mathrm{~m}^{-3}$. In ion ITB plasma, $\chi_{i}$ normalized by $T_{i}^{1.5}$ reduces [30]. The normalization factor $T_{i}{ }^{1.5}$ is temperature dependence of gyro-Bohm scaling. The improvement after normalization becomes higher towards the central region of the plasma. Thus, this confinement mode is called ion ITB.

The other plasma is high density low power, which is so-called "normal confinement plasma". The set of normal confinement plasma in Fig.1 were heated by a 7.5MW NB, which were $3 \mathrm{MW}$ perp-PNB and $4.5 \mathrm{MW}$ para-NNB. The line averaged density was $2.9 \times 10^{19} \mathrm{~m}^{-3}$. The data sets of normal confinement plasma are comparable dataset of the LHD database for ISS04. As shown in Fig.1 (a), with increase of He concentration, a clear increase of $T_{i}$ is found in ion-ITB plasma, while modest increase of $T_{i}$ is observed in normal confinement plasma. As shown in Fig.1 (b), modest increase of the $T_{e}$ is found with increase of He concentration both in ion ITB and normal confinement plasma. This 
suggests improvement of the ion energy confinement occurs with increases of He concentration only in ion ITB plasma. However, detail transport analyses are necessary taking into account difference of ion density and deposition power.

In the following subsections, profiles of the typical set of normal and ion ITB plasma are compared. The characteristics of measured ion scale turbulence are described. Then, result of power balance analyses are shown and comparison with of neoclassical estimation are described.

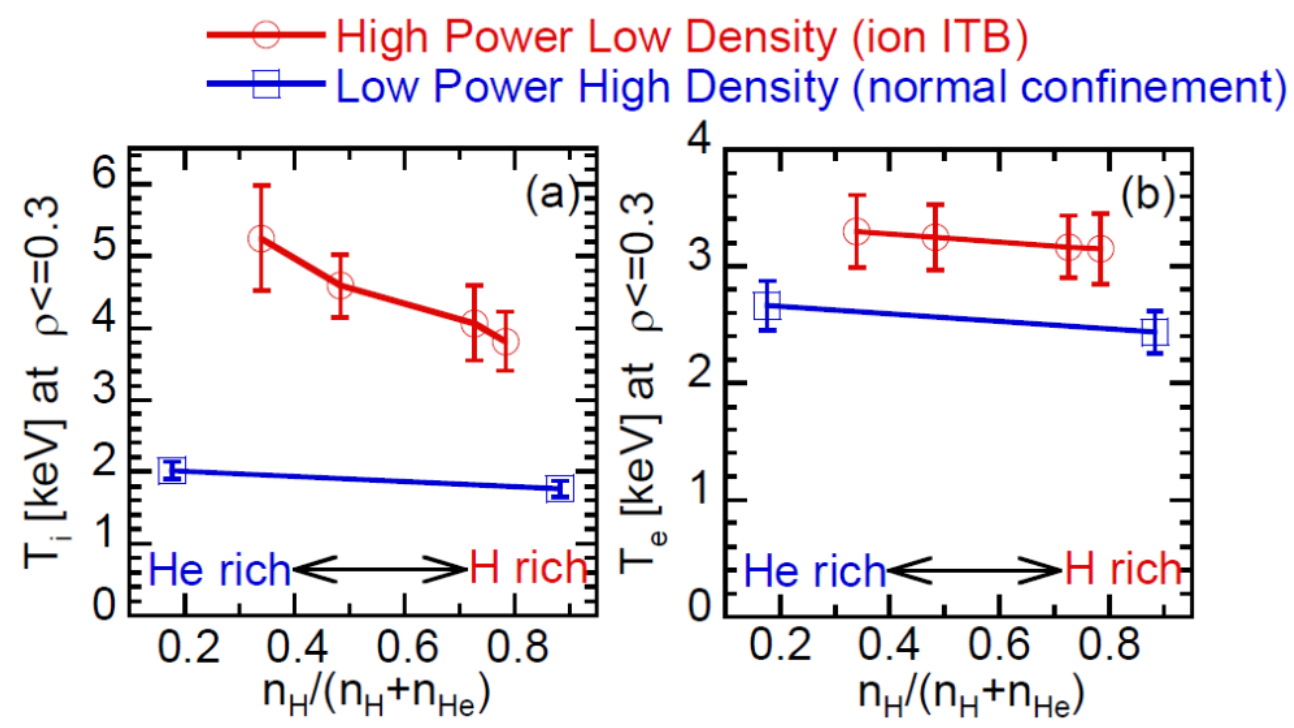

FIG.1. Comparison achieved (a) central ion and (b) electron temperatures in $\mathrm{H}$ and He rich plasma. High power low density plasma (ion ITB plasma) is PNB=20-23MW, ne bar $=1.3 \times 10^{19} \mathrm{~m}^{-3}$. Low power high density plasma (normal confinement plasma) is $P N B=$ 7-7.5MW, ne_bar $=2.9 \times 10^{19} \mathrm{~m}^{-3}$.

\subsection{Profiles and energy transport characteristics of normal confinement plasma}

We begin describing the chracteristics of the normal confinement plasma, since this dataset is standard dataset. Figure 2 shows profiles of $\mathrm{He}$ and $\mathrm{H}$ rich plasma of normal confinement plasma. The two shots are data of normal confinement plasmas in Fig.1.

In Fig.2 (a-1) and (b-1), electron density profiles were measured by YAG laser Thomson scattering (YAG TMS) [31]. The electron density profiles were cross-checked by Abel inversion profiles of far-infrared laser interferometer [32] and showed reasonable agreements [33]. In Fig.2 (a-1) and (b-1), ion density profiles are also shown. The ion density is sum of $\mathrm{H}^{+}, \mathrm{He}^{2+}$ and $\mathrm{C}^{6+}$ ions. The $\mathrm{C}^{6+}$ profiles were directly measured by charge exchange spectroscopy (CXRS) [34]. $\mathrm{H}^{+}$and $\mathrm{He}^{2+}$ profiles are estimated from $\mathrm{n}_{\mathrm{e}}$ profile, $\mathrm{C}^{6+}$ profile and $\mathrm{n}_{\mathrm{H}} /\left(\mathrm{n}_{\mathrm{H}}+\mathrm{n}_{\mathrm{He}}\right)$. $\mathrm{n}_{\mathrm{H}} /\left(\mathrm{n}_{\mathrm{H}}+\mathrm{n}_{\mathrm{He}}\right)$ is assumed to be spatially constant. As shown in Fig.2 (a-1) and (b-1), $\mathrm{n}_{\mathrm{e}}$ profiles are similar in He and $\mathrm{H}$ rich plasma. However, 
$\mathrm{n}_{\text {ion }}$ is different and $\mathrm{n}_{\text {ion }}$ in He rich plasma is about $60 \%$ of $\mathrm{n}_{\text {ion }}$ in $\mathrm{H}$ rich plasma. Electron and ion density profiles are slightly hollow. This is widely observed in LHD [13].

Figure $2(\mathrm{a}-2)$ and (b-2) shows $\mathrm{T}_{\mathrm{e}}$ profile measured by YAG TMS and $\mathrm{T}_{\mathrm{i}}$ profiles measured by CXRS. $\mathrm{T}_{\mathrm{e}}$ and $\mathrm{T}_{\mathrm{i}}$ are slightly higher in He rich than in $\mathrm{H}$ rich plasma. In both cases, $T_{e}$ is higher than $T_{i}$ at $\rho<0.8$ and almost same at $\rho=0.8-1.0$. The positive $\rho$ of $n_{e}$, $\mathrm{T}_{\mathrm{e}}$ and $\mathrm{T}_{\mathrm{i}}$ profiles correspond to the torus inner side of the magnetic axis in the horizontally elongated cross section, negative $\rho$ to torus outer side. These profiles are almost identical at positive and negative $\rho$.

Figure 2 (a-3) (a-5) and (b-3) (b-5), are fluctuation profiles measured by two dimensional phase contrast imaging (2D-PCI) [35]. A detailed analysis scheme is described in ref. 36. The 2D-PCI measures the turbulence of $\mathrm{k}=0.1-0.8 \mathrm{~mm}^{-1}$ corresponding to $\mathrm{k} \rho_{\mathrm{i}} \sim 0.1-2, \mathrm{f}=20-500 \mathrm{kHz}$ at $\rho>\sim 0.3$. The $\mathrm{CO}_{2}$ laser beam passes the vertically elongated cross section and measures turbulence from both the upper side indicated by positive $\rho$ and the lower side indicated by negative $\rho$ of relative to the equatorial plane. The wavenumber is poloidally dominated. The sign of $\mathrm{k}$ propagating in the same poloidal direction (electron diamagnetic or ion diamagnetic direction) of upper and lower side shows opposite sign [35]. The phase velocity in laboratory frame can be measured from k-f relation. In Fig.2, fluctuation profiles were obtained for the data of 80 msec of $1 \mu \mathrm{sec}$ sampling data.

As shown in Fig.2 (a-3) and (b-3), fluctuation amplitudes have peaks at abs( $\rho) \sim 1.0$. Fluctuation amplitude is smaller in He rich than in $\mathrm{H}$ rich plasma. As shown in Fig.2 (a4) and (b-4), k spectrum is similar in both cases and most of the contribution is less than $0.4 \mathrm{~mm}^{-1}$. The ion Larmor radius is $0.8 \mathrm{~mm}$ at $\rho=1$. Thus, the upper limit of the spectrum is $k \rho_{i}=0.32$. As shown in Fig. $2(a-5)$ and (b-5), the turbulence propagates towards the electron diamagnetic direction in laboratory frame. The phase velocity of the tuburbulence is close to the $\mathrm{E}_{\mathrm{r}} \mathrm{XB}_{\mathrm{t}}$ poloidal rotation velocity measured by CXRS. 


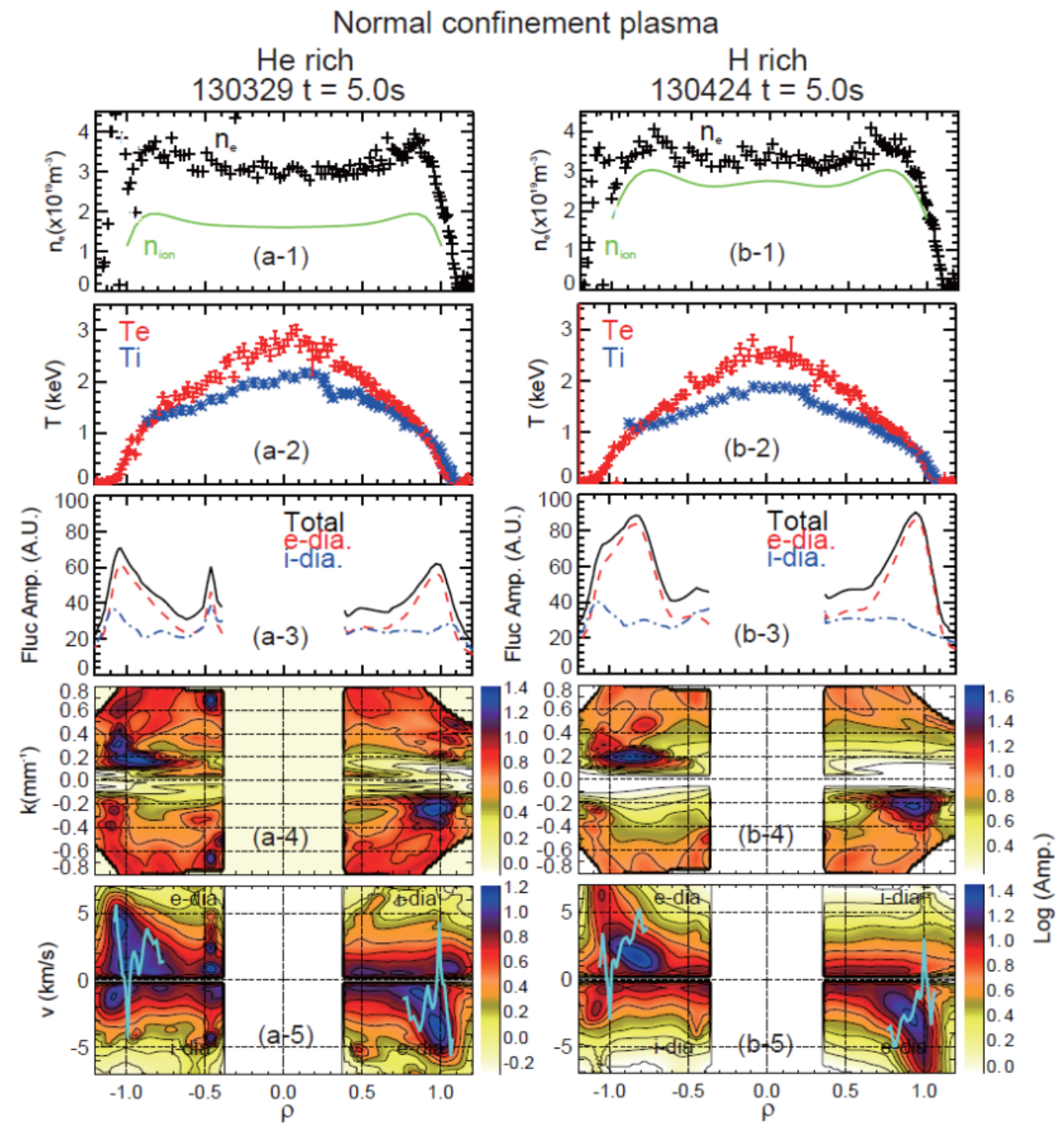

FIG. 2 Profiles in He rich (a-1) (a-5) and H-rich (b-1) (b-5) of normal confinement plasmas. $(a-1),(b-1) n_{e}$ (symbols) and $n_{\text {ion }}$ profile (green lines), (a-2),(b-2) $T_{e}$ and $T_{i}$ profiles, (a-3),(c-3) electron density fluctuation amplitude, (a-4), (d-4) contour plot of fluctuation amplitude $k$ spectrum and (a-5), (b-5) contour plot of fluctuation amplitude phase velocity in laboratory frame. In (a-5), (b-5), blue lines indicate $E_{r} x B_{t}$ poloidal rotation velocities measured by CXRS.

The power balance analyses were carried out by using TASK3D-a code [37], which takes into account multi ion species. The results were compared with neoclassical prediction. Neoclassical $E_{r}$ and transport coefficients were calculated by GSRAKE [38]. GSRAKE employs the bounce-average approach of drift kinetic equation. Presently GSRAKE does not include multi ion species and the effects of the external torque input. The external torque, which can be induced by the tangentially injected NBI, can affect neoclassical transport. The co- $\mathrm{B}_{\mathrm{t}}$ dominant NBI can induce positive $\mathrm{E}_{\mathrm{r}}$, then neoclassical 
coefficients become lower [39]. However, in normal confinement of Fig.2, the effects of external torque input is weak, because unbalanced external torque by tangentially injected NBI is small. Present calculation will provide a brief idea of the neoclassical transport level because of the above mentioned limitations.

Figure 3 shows experimental $E_{r}, \chi_{i}, \chi_{e}$ and comparison with neoclassical estimation. Due to the diagnostic limit of CXRS, the reliable $E_{\mathrm{r}}$ data is presently available only at $\rho>0.7$. Experimental $\mathrm{E}_{\mathrm{r}}$ is almost identical in both cases. Considering the error bars, both datasets are not highly inconsistent with neoclassical estimations. Also, experimental $\mathrm{E}_{\mathrm{r}}$ well agrees with neoclassical prediction. At $\rho<0.8$, Er is negative indicating ion root.

Experimental $\chi_{i}$ is higher in He rich plasma at $\rho<0.8$ than in $\mathrm{H}$ rich plasma and almost identical at $\rho>0.8$. It should be noted that $\mathrm{T}_{\mathrm{i}}$ is slightly higher in He rich than in $\mathrm{H}$ rich plasma as shown in Fig.1 (a), Fig.2 (a-2) and (b-2). However, the ion density is lower in He rich plasma than in $\mathrm{H}$ rich plasma as shown in Fig.2 (a-1) and (b-1). Thus, the higher $\mathrm{T}_{\mathrm{i}}$ in He rich plasma is not due to the reduced ion trasnport but to the higher deposition power per ion.

The experimental $\chi_{\mathrm{i}}$ at $\rho<0.8$ is around a factor 1.2 2 higher than neoclassical values in both $\mathrm{He}$ and $\mathrm{H}$ rich plasma. Neoclassical contribution becomes smaller in both case at $\rho>0.8$, where ion scale turbulence exists as shown in Fig.2 (a-3) (b-5).

The experimental $\chi_{\mathrm{e}}$ is almost identical at $\rho<0.5$ in both cases. Towards the plasma boundary, experimental $\chi_{\mathrm{e}}$ becomes lower in He rich plasma. This is due to the steeper $\mathrm{T}_{\mathrm{e}}$ gradient in He rich plasma. In both $\mathrm{He}$ and $\mathrm{H}$ rich plasmas, experimental and neoclassical $\chi_{\mathrm{e}}$ are comparable at $\rho<0.8$. Neoclassical contribution of $\chi_{\mathrm{e}}$ becomes lower toward the plasma boundary as well as $\chi_{i}$ However, neoclassical contribution of $\chi_{\mathrm{e}}$ is much smaller than $\chi_{\mathrm{i}}$ due to the increasing experimental $\chi_{\mathrm{e}}$ toward the plasma boundary.

Also, it should be noted that experimental and neoclassical $\chi_{i}$ is higher than $\chi_{\mathrm{e}}$ in both $\mathrm{He}$ and $\mathrm{H}$ rich cases. 

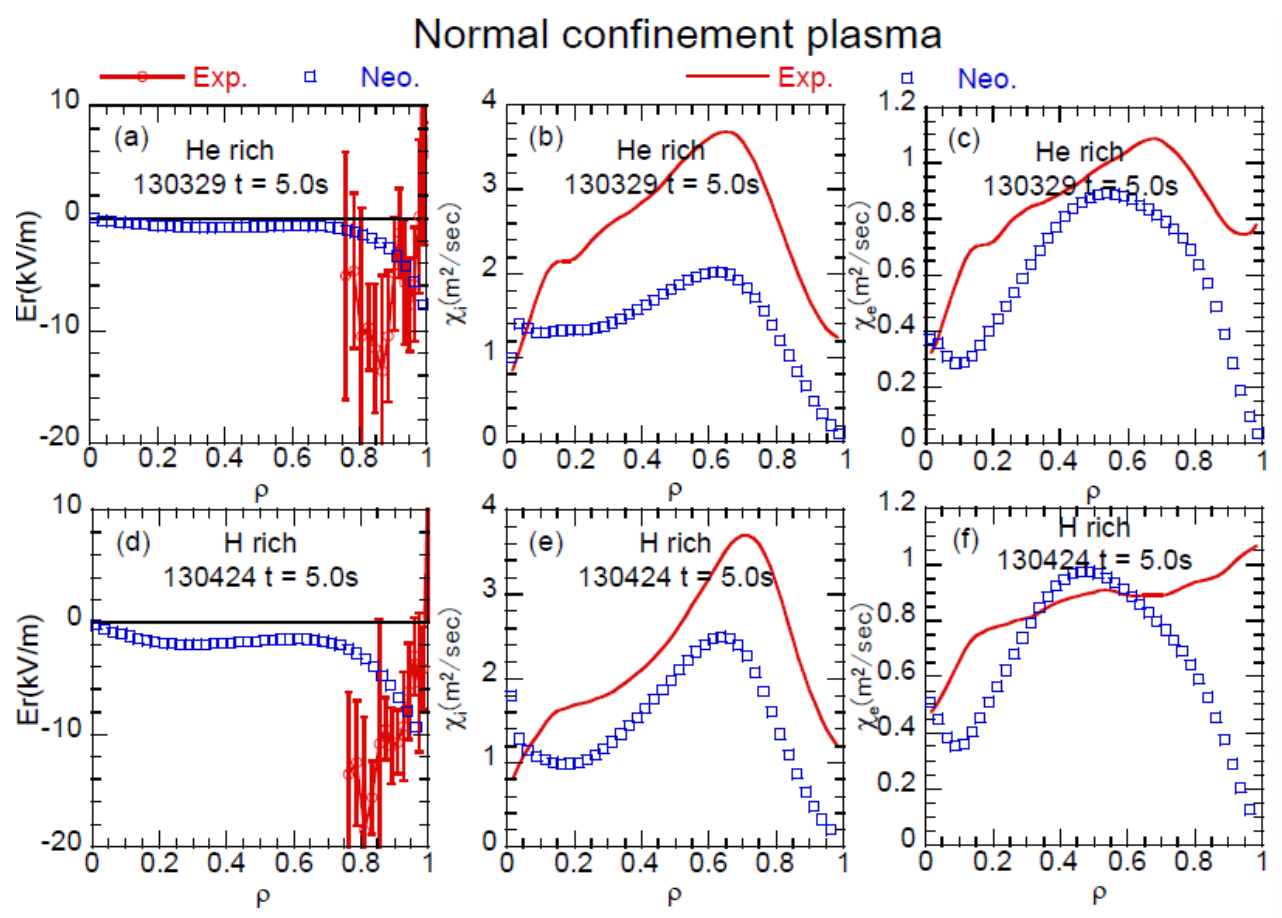

FIG.3 Comparison with neoclassical estimation in normal confinement plasma. (a),(d) $E_{r}$, (b), (e) $\chi_{i}$ and (c), (f) $\chi_{e}$. Same shot and timing as Fig. 2. Same shot and timing as Fig. 2. (a) (c) in He rich, (f) f), H rich. Neoclassical estimation of 130329 was estimated for pure He and 130424 for pure $H$.

\subsection{Profiles and energy transport characteristics of ion ITB plasma}

In this section, we describe the characteristics of the ion ITB plasma from profile, power balance analysis and neoclassical estimations.

Figure 4 shows profiles in ion ITB plasmas. Figure 4 are data of the highest and lowest $\mathrm{T}_{\mathrm{i}}$ and He concentration of ion ITB plasma in Fig.1. As shown in Fig.4 (a-1), (b-1), electron and ion density profiles are slightly hollow. This is similar profiles in as normal confinement plasma. The shape of density profiles are not very different from ones in normal confinement. As shown in Fig.4 (a-2) and (b-2), $\mathrm{T}_{\mathrm{e}}$ profiles are almost identical in $\mathrm{He}$ and $\mathrm{H}$ rich plasma, while $\mathrm{T}_{\mathrm{i}}$ is clearly higher in He rich plasma.

The time averaged fluctuation profiles are almost identical both in $\mathrm{He}$ and $\mathrm{H}$ rich plasmas. The peak position of fluctuation is around $\rho=0.7$. The fluctuation has asymmetry in positive and negative $\rho$ (upper and lower side of equatorial plane). One of the possible interpretations of the asymmetry is turbulence eddy tilting. This is possibly due to the formation of $E_{r}$ shear. Such asymmetry was observed in ion ITB plasma with carbon pellet injection [41] and recent results of simultaneous achievements of high $T_{e}$ and $\mathrm{T}_{\mathrm{i}}[42]$. 
The $\mathrm{k}$ spectrum is less than $0.4 \mathrm{~mm}^{-1}$. The ion Larmor radius is about $1.7 \mathrm{~mm}$ at $\rho=0.7$. Thus, the upper limit of spectrum is $k \rho_{i}=0.68$. The fluctuation propagates towards the ion diamagnetic direction in laboratory frame and phase velocity is comparable to the $\mathrm{E}_{\mathrm{r}} \mathrm{xB}$ poloidal rotation velocities.

The difference of time averaged fluctuation profiles are very similar in $\mathrm{He}$ and $\mathrm{H}$ rich plasma in spite of clear difference of $\mathrm{T}_{i}$ profiles, however, these profiles are very different from the profiles in normal confinement plasmas.

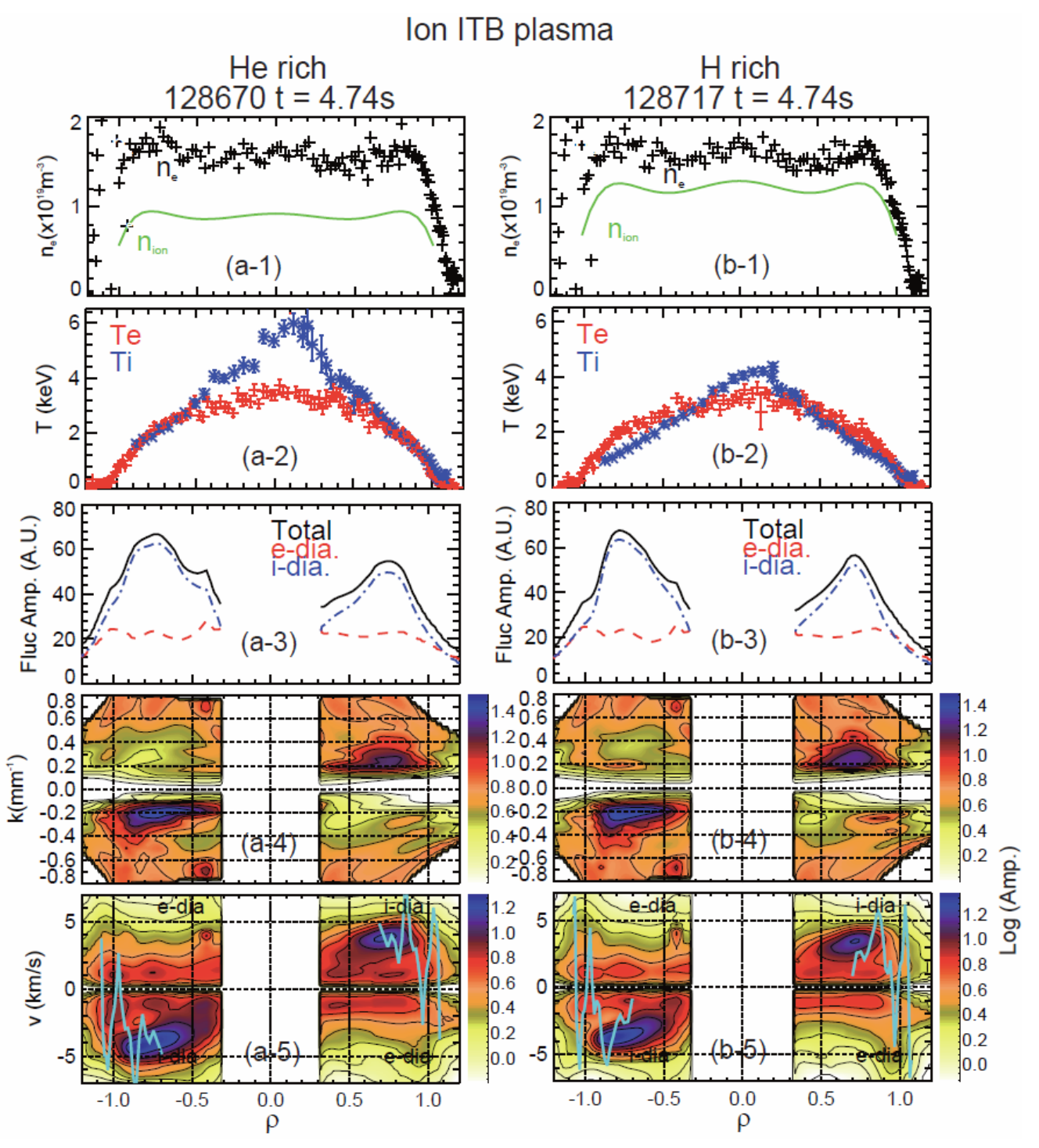

FIG.4 Profiles in He rich (a-1) (a-5) and H-rich $(b-1) \sim(b-5)$ of ion ITB plasmas.

(a-1),(b-1) $n_{e}$ (symbols) and $n_{\text {ion }}$ profile (green lines), (a-2),(b-2) $T_{e}$ and $T_{i}$ profiles, (a-3), (c3) electron density fluctuation amplitude, $(a-4),(d-4)$ contour plot of fluctuation amplitude $k$ spectrum and (a-5), (b-5) contour plot of fluctuation amplitude phase velocity in laboratory 
frame. In (a-5), (b-5), blue lines indicate ExB poloidal rotation velocities measured by CXRS.

Figure 5 shows experimental $E_{r}, \chi_{i}, \chi_{e}$ and comparison with neoclassical estimation of ion ITB plasma. As shown in Fig.5 (a) and (d), the experimental $\mathrm{E}_{\mathrm{r}}$ is almost identical in $\mathrm{He}$ and $\mathrm{H}$ rich plasmas. Both electron and ion roots are theoretically possible at $\rho>8$. The experimental $E_{r}$ well agrees with neoclassical prediction of electron root at $\rho>0.8$. At $\rho<0.8$, only ion root is possible. However, as shown in Fig. 4 (a-5) and (b-5), phase velocity of the turbulence propagates towards the ion diamagnetic direction at $\rho=0.4 \sim$ 1.0. This observation suggests that $E_{\mathrm{r}}$ is also positive at this radial locations. Thus, it is likely that there are electron roots at $\rho<0.8$ against GSRAKE prediction. In the ion ITB plasmas, three tangential NBs (total $11.5 \mathrm{MW}$ ) and two perpendicular NBs (total $11.5 \mathrm{MW})$ were injected. Two of NBs (7.5MW) were co-Bt directed and one of NBs (4MW) was counter-Bt directed. Then, a 3.5MW was co-dominant injection. Such a codominant injection induces positive $\mathrm{E}_{\mathrm{r}}$ and reduce neoclassical transport. Thus, neoclassical estimation of ion root at $\rho<0.8$ is likely to be overestimated.

As shown in Fig. 5 (b) and (e), $\chi_{i}$ in He rich is lower than in $\mathrm{H}$ rich plasma at almost the entire region of the plasma. This indicates higher $T_{i}$ in He rich plasma is not due to the larger deposition per ion due to lower ion density but is due to the lower transport. This is an important contrast to the normal confinement plasmas.

The electron root $\chi_{i}$ at $\rho>0.8$ is $10 \sim 30 \%$ of experimental $\chi_{i}$ both in $\mathrm{He}$ and $\mathrm{H}$ rich plasmas. At $\rho<0.8$, experimental $\chi_{i}$ is comparable with neoclassical $\chi_{i}$. However, as mentioned above, neoclassical $\chi_{\mathrm{i}}$ can be smaller due to the co-dominant toroidal torque.

As shown in Fig.5 (c) and (f), the experimental $\chi_{\mathrm{e}}$ is higher in He rich plasma than in $\mathrm{H}$ rich plasma. Both in $\mathrm{He}$ and $\mathrm{H}$ rich plasma, experimental $\chi_{\mathrm{e}}$ increases toward the plasma center. The electron root $\chi_{\mathrm{e}}$ at $\rho>0.8$ is comparable with experimental $\chi_{\mathrm{e}}$. This indicates anomalous contribution of electron transport is smaller than that of ion transport. As mentioned above, neoclassical $\chi_{\mathrm{e}}$ at $\rho<0.8$ is likely to be overestimated. However, experimental $\chi_{\mathrm{e}}$ is clearly exceeds the neoclassical values. Anomalous contribution of electron transport increases towards the plasma center. The increasing $\chi_{\mathrm{e}}$ towards the plasma center is due to the decreasing $\mathrm{T}_{\mathrm{e}}$ gradient towards the plasma center. Fluctuation becomes smaller towards the center, thus, the results suggests that turbulence, which is not measured (for example electron scale turbulence), may play role on anomalous transport in the central region. 


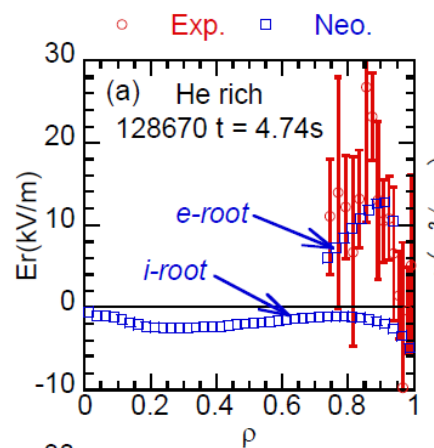

Ion ITB plasma
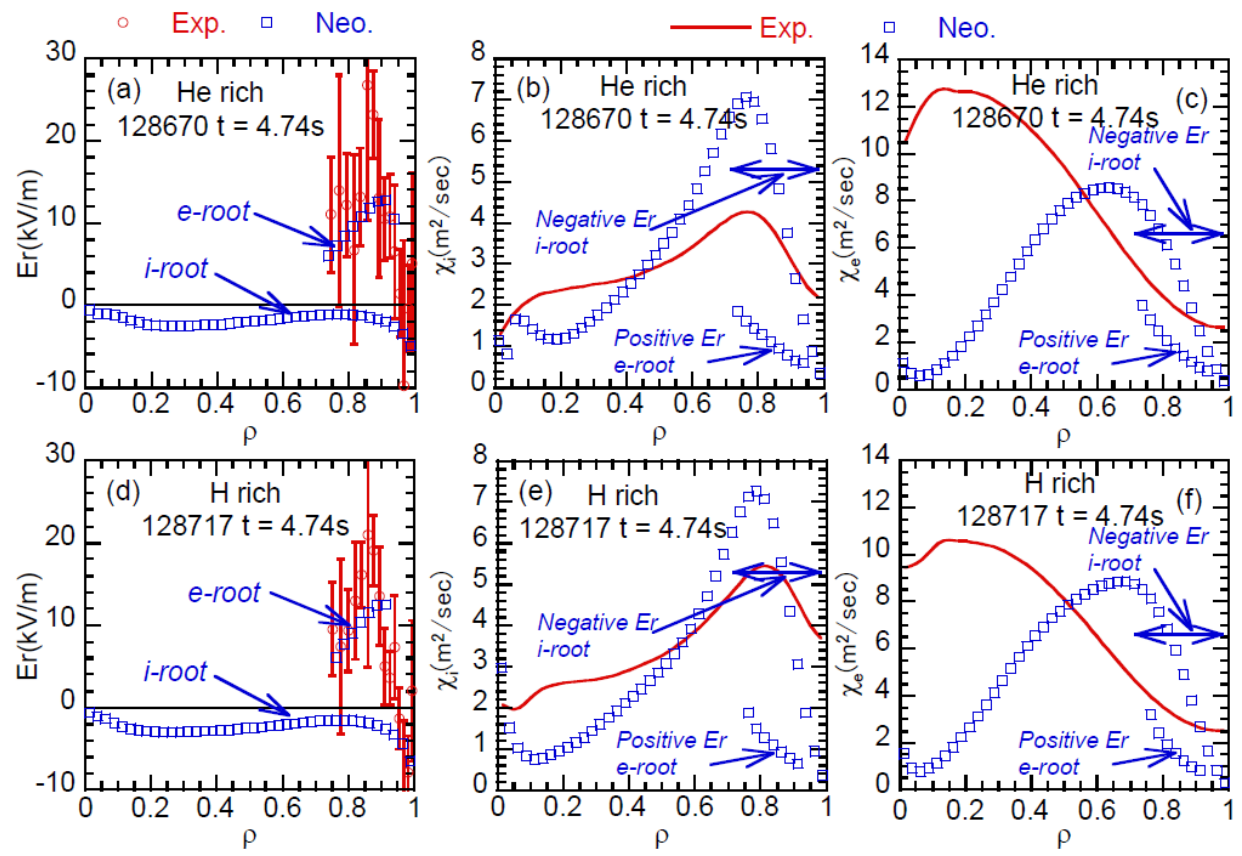

FIG.5 Comparison with neoclassical estimation in ion ITB plasma. (a), (d) $E_{r},(b),(e) \chi_{i}$ and (c), (f) $\chi_{e}$. Same shot and timing as Fig. 2. (a) (c) in He rich, (f) f), H rich. Same shot and timing as Fig. 4. Neoclassical estimation of 128670 was estimated for pure He and 128717 for pure $H$.

\subsection{Confinement improvement in He rich plasma}

As described in the previous section, higher $\mathrm{T}_{\mathrm{i}}$ in He rich plasma of normal confinement is higher deposition per ion. Higher $T_{i}$ in He rich plasma of ion ITB is reduced ion transport. Here we compare $T_{i}$ and fluctuation profile again and compare $\chi_{i}$ with gyroBohm prediction for a more precise argument about confinement improvement.

\subsubsection{Profile stiffness of ion temperature profile and role of turbulence}

Figure $6(\mathrm{a}-1)$ and (b-1) shows comparison of $\mathrm{T}_{\mathrm{i}}$ profiles in normal confinement and ion ITB. The vertical axes are logarithmic scale. Thus, the gradient in the figure shows the normalized $\mathrm{T}_{\mathrm{i}}$ gradient $\left(\operatorname{grad} \mathrm{T}_{\mathrm{i}} / \mathrm{T}_{\mathrm{i}}=\mathrm{L}_{\mathrm{Ti}}{ }^{-1}\right.$ (i.e., inverse of $\mathrm{T}_{\mathrm{i}}$ scale length) ).

In Fig. 6 (a-1) and (b-1), one clear characteristic of the $\mathrm{T}_{\mathrm{i}}$ profiles is the change of $\mathrm{L}_{\mathrm{Ti}}{ }^{-1}$ at $\rho=0.9$. This is seen both in normal and ion ITB plasma and in He and $\mathrm{H}$ rich plasma. There is an edge pedestal shoulder of $\mathrm{T}_{i}$ profile similar to tokamak $\mathrm{H}$ mode.

As shown in Fig. 6 (a-1), in normal confinement plasmas, $T_{i}$ at pedestal top (at $\rho \sim 0.9$ ) is higher in $\mathrm{He}$ rich than in $\mathrm{H}$ rich plasmas but, $\mathrm{L}_{\mathrm{Ti}}^{-1}$ at $\rho<\sim 0.9$ in He rich plasma is lower. Then, $\mathrm{T}_{\mathrm{i}}$ of central region in He rich becomes close to central $\mathrm{T}_{\mathrm{i}}$ in $\mathrm{H}$ rich plasma in spite of higher $T_{i}$ edge pedestal. 
As shown in Fig.6 (b-1), in ion ITB plasma, $T_{i}$ at pedestal top (at $\rho \sim 0.9$ ) is higher in $\mathrm{He}$ rich than in $\mathrm{H}$ rich plasmas as in the case for normal confinement. However, $\mathrm{L}_{\mathrm{Ti}}{ }^{-1}$ at $\rho$ $<\sim 0.9$ in $\mathrm{He}$ and $\mathrm{H}$ rich plasma are similar. Then, higher edge pedestal in He rich induces clearly higher central $T_{i}$. These observations suggest that $T_{i}$ profiles are stiff in ion ITB plasmas.

The characteristics of the stiffness can be linked with turbulence threshold. As shown in Fig. 6 (b-2), in ion ITB, most of the fluctuation components exist inside the edge pedestal (at $\rho<0.9$ ). This fluctuation possibly determines the stiff $\mathrm{L}_{\mathrm{Ti}}{ }^{-1}$. It is an important contrast that in normal confinement, at $\rho<0.9$, fluctuation becomes small as shown in Fig.6 (a2). This is possible reason why $\mathrm{L}_{\mathrm{Ti}}{ }^{-1}$ is not stiff in normal confinement.

Gyrokinetic linear analyses were performed by using the local flux tube gyrokinetic code GS2 $[43,44]$ for the further investigation of $T_{i}$ stiffness in ion ITB plasmas. Figure 7 (a) shows linear spectrum of growth rate $(\gamma)$ and real frequency $\left(\omega_{\mathrm{r}}\right)$ at $\rho=0.7$, which is the peak position of the fluctuation, in $\mathrm{He}$ and $\mathrm{H}$ rich ion ITB plasmas. Electrostatic approximation was made. The kinetic effects of four species (electron, $\mathrm{H}+, \mathrm{He} 2+$ and C6+) were calculated including collision effects among the species. In both cases, the dominant instabilities are ion temperature gradient (ITG) mode. As shown in Fig.7(a), the growth rate is lower in He rich plasma. This is due to the lower $\mathrm{T}_{\mathrm{e}} / \mathrm{T}_{\mathrm{i}}$ and higher $\mathrm{He}^{2+}$ concentration.

The linear thresholds were investigated scanning $\mathrm{L}_{\mathrm{Ti}}{ }^{-1}$ and keeping other parameters constant. As shown in Fig.7 (b), the linear ITG threshold is almost the same $\left(\mathrm{L}_{\mathrm{Ti}}{ }^{-1}=2.6 \mathrm{~m}^{-}\right.$ ${ }^{1}$ ) in He- and H-rich plasmas. The experimental $\mathrm{L}_{\mathrm{Ti}}{ }^{-1}$ is $3.3 \mathrm{~m}^{-1}$ in He rich and $2.8 \mathrm{~m}^{-1}$ in $\mathrm{H}$ rich. They are within factor 1.3 of linear threshold. ITG threshold likely determines $\mathrm{L}_{\mathrm{Ti}^{-1}}{ }^{-1}$ inside of pedestal shoulder. Thus, higher edge pedestal $\mathrm{T}_{\mathrm{i}}$ in He rich can induce higher central $T_{i}$ due to the $T_{i}$ profile stiffness. 

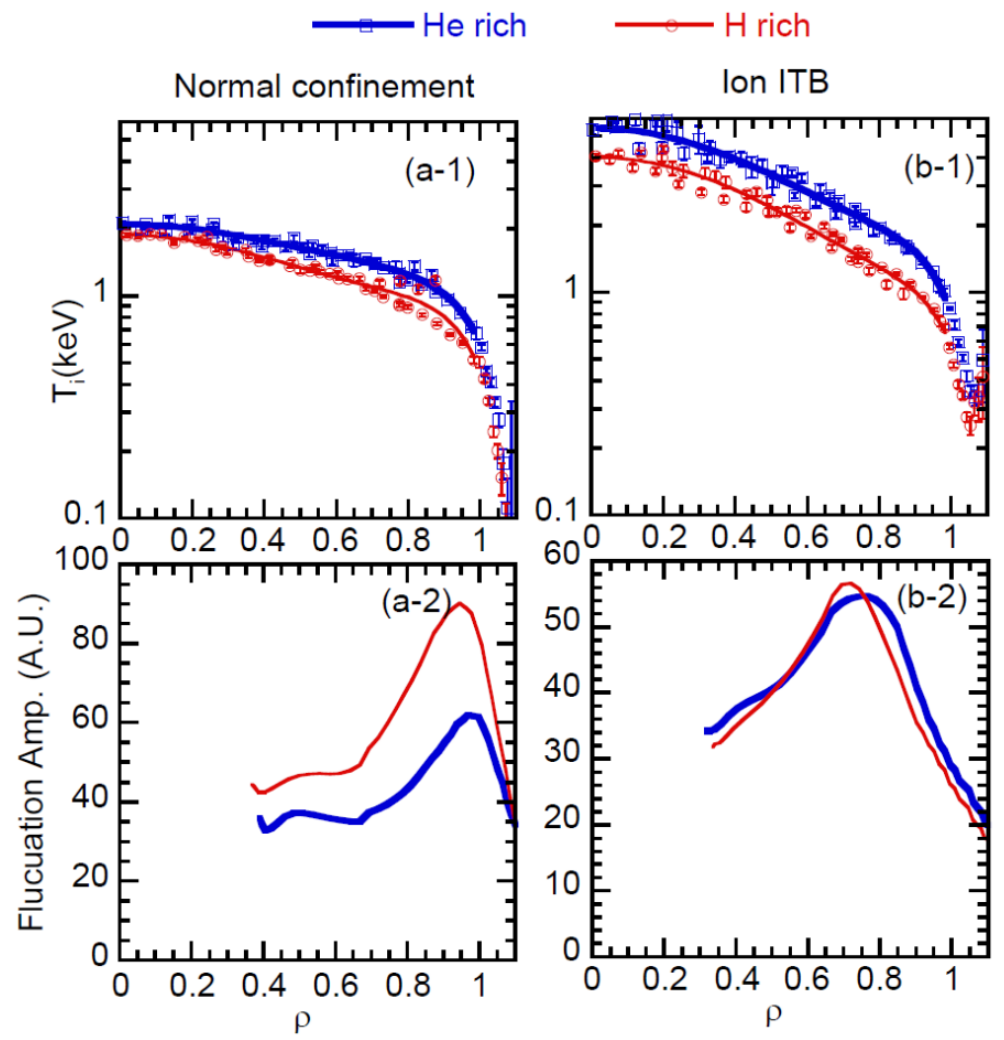

FIG.6 Comparison of $T_{i}$ and fluctuation profiles (a-1), (b-1) $T_{i}$ profiles and (b-1) and (b-2) fluctuation profiles.

(a-1),(a-2) in normal confinement plasma, and (b-1), (b-2) ion ITB plasma. The Vertical axes of (a-1) and (b-1) are logarithmic scale.

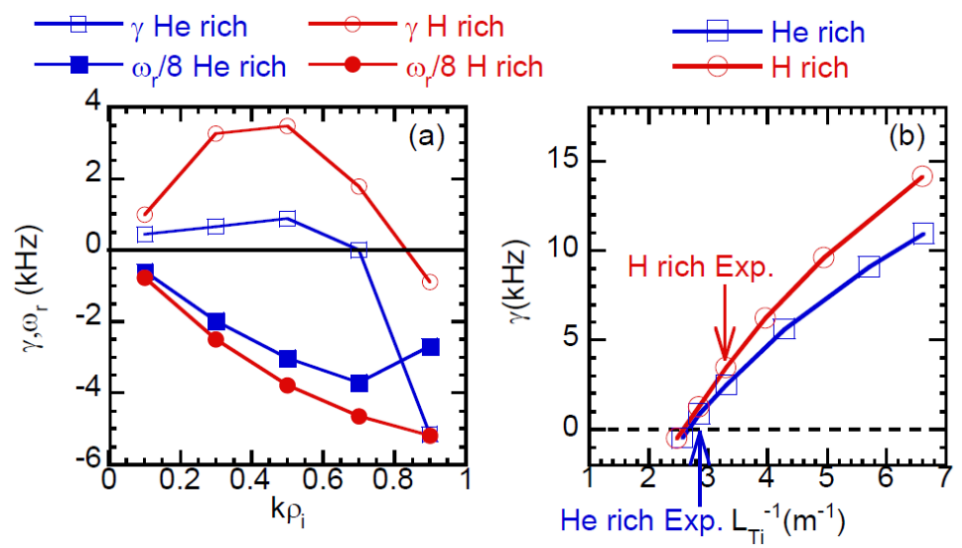

FIG.7 (a) Linear spectrum at $\rho=0.7$, (b) $L_{T i}^{-1}$ dependence of $k \rho_{i}=0.5$ at $\rho=0.7$. Negative $\omega_{r}$ indicates ion diamagnetic direction

\subsubsection{Comparison with gyro-Bohm prediction}

As described in Section 2.1 and 2.2, higher $T_{i}$ in He rich normal confinement plasma is 
due to larger deposition power per ion and higher $T_{i}$ in He rich ion ITB plasma is due to the reduced transport. However, confinement improvement should be argued by normalization using a reference. For the normalization, we used gyro-Bohm factor. This is due to the following reasons. First, ISS04 scaling follows the gyro-Bohm scaling. Second, linear growth rate of ITG/TEM turbulence follows gyro-Bohm factor [45, 46, 47].

The gyro-Bohm factor is defined by $\mathrm{m}_{\mathrm{i}}^{0.5} \mathrm{~T}_{\mathrm{i}}{ }^{1.5} /\left(\mathrm{aq}^{2} \mathrm{~B}_{\mathrm{t}}{ }^{2}\right)$, where $\mathrm{m}_{\mathrm{i}}$ is mass, $\mathrm{T}_{\mathrm{i}}$ is ion temperature, $q$ is the charge of each charged particle, a is minor radius, and $B_{t}$ is the magnetic field. The discrepancy from the gyro-Bohm parameter dependence is an indicator of improvement or deterioration of confinement. It should be noted that dependence on $\mathrm{q}$ is usually not considered in the conventional scaling. However, $\mathrm{q}$ has strong effects in the gyro-Bohm diffusivity and is necessary to take into account. Here, we defined the gyro-Bohm factor as $\mathrm{F}_{\mathrm{GB}}=\mathrm{m}_{\text {ieff }}{ }^{0.5} \mathrm{~T}_{\mathrm{i}}{ }^{1.5} /\left(\mathrm{q}_{\text {ieff }}{ }^{2}\right)$, where $\mathrm{m}_{\text {ieff }}$ is effective ion mass and $q_{i e f f}$ is effective ion charge. $m_{\text {ieff }}$ and $q_{i e f f}$ were estimated from the ratio of the ions. The experiments were performed at $\mathrm{B}_{\mathrm{t}}=2.75 \mathrm{~T}$ and $\mathrm{R}_{\mathrm{ax}}=3.6 \mathrm{~m}$. The effects of $\mathrm{B}$ and $\mathrm{a}$ are neglected. For the same temperature and the magnetic field, $\mathrm{F}_{\mathrm{GB}}$ of pure He plasma is $50 \%$ of that of pure $\mathrm{H}$ plasma. Thus, it is reasonable if transport in He rich plasma is smaller than that in $\mathrm{H}$ rich plasma.

Figure 8 shows a comparison of normalized $\chi_{i}$, which is defined by $\chi_{i} / F_{G B}$. Figure 8 show clear contrast in normal confinement and ion ITB plasma. In normal confinement, $\chi_{\mathrm{i}} / \mathrm{F}_{\mathrm{GB}}$ in He rich is higher plasma at $\rho<0$.8. In ion ITB, $\chi_{\mathrm{i}} / \mathrm{F}_{\mathrm{GB}}$ is comparable at $\rho<0.6$ and $\chi_{i} / F_{G B}$ in He rich is higher at $\rho>0.6$. The reduction of $\chi_{i} / F_{G B}$ in He rich becomes more significant toward the plasma boundary. The followings are conclusion about the confinement improvement relative to gyro-Bohm prediction.

In normal confinement, ion confinement is degraded in He rich plasma. The degradation is more significant toward the plasma center. In ion ITB, ion confinement is improved in He rich plasma. The improvement is more significant toward the plasma boundary. Confinement status and improvement/deterioration location is different in normal confinement and ion ITB plasma. 

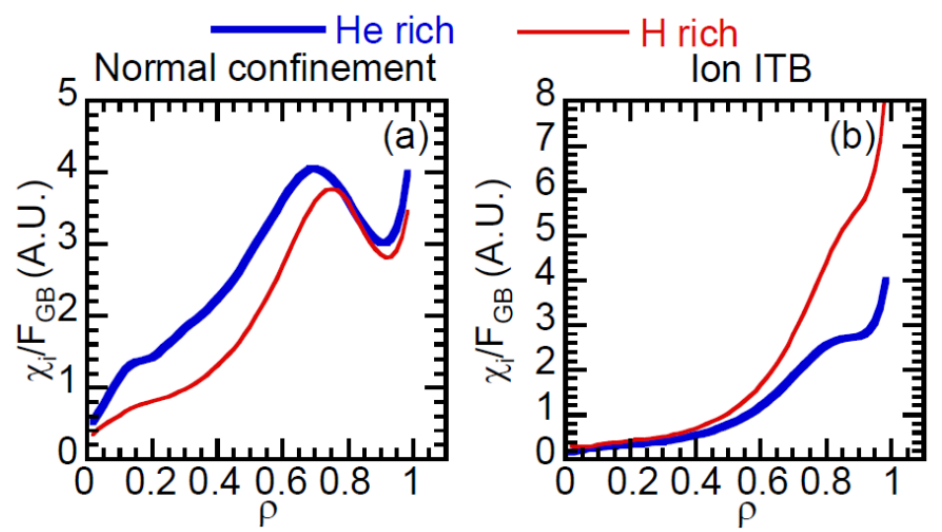

FIG.8 Comparison of normalized $\chi_{i}\left(\chi_{i} / F_{G B}\right)(a)$ in normal confinement plasma and $(b)$ ion ITB plasma.

\section{Parameter dependence of heat transport coefficients}

In the previous section, transport characteristics were investigated in detail in the normal confinement and ion ITB plasma. In this section, parameter dependence of $\chi_{\mathrm{i}}$ and $\chi_{\mathrm{e}}$ are investigated. Firstly, we investigate the collisionality dependence. Collisionality can influence both neoclassical and anomalous transport. In the data set, magnetic field was fixed at $2.75 \sim 2.85 \mathrm{~T}$. Thus, beta and normalized ion radius do not vary very much, while collisionality varies more than one order of magnitude. The present data set is good database of collisionality scan. As described in Section 2, characteristics of transport in the normal confinement and ion ITB plasma is common both in He and $\mathrm{H}$ rich plasma. This indicates that collisionality play a crucial role, while the effects of the ion species are secondary. Thus, collisionality dependence of $\chi_{\mathrm{e}}$ and $\chi_{\mathrm{i}}$ are surveyed, and then the effects of ion species are investigated

Figure 9 shows the normalized collisionality $\left(v_{h}{ }^{*}\right)$ dependence of at $\rho=0.3,0.5,0.7$ and 1.0. Here $v_{\mathrm{h}}{ }^{*}$ is defined as $v^{*}{ }_{\mathrm{h}}=v_{\mathrm{ei}} /\left(\varepsilon_{\mathrm{eff}}{ }^{3 / 2} v_{\mathrm{T}} / q R_{m j}\right) . v_{e i}$ is the electron ion collision frequency, $v_{\mathrm{T}}$ is the electron thermal velocity, $q$ is the safety factor, $R_{m j}$ is the major radius, and $\varepsilon_{\mathrm{eff}}$ is an effective helical ripple $[48,49] . v^{*}{ }_{h}=1$ corresponds to the boundary of $1 / v$ and plateau regime in helical/stellarator [13], which corresponds to approximately banana regime and plateau regime in tokamak with the same aspect ratio of LHD. In Fig.9, power balance analyses were performed for 95 cases from 21 shots. These shots data were obtained in the 2011 and the 2014 campaigns. In order to check the reproducibility of the analyses, four to five timings were analyzed for the almost steady state of each shot. Heating power and line averaged density were scanned to vary $v^{*}{ }_{h}$. The concentration ratio was scanned from $\mathrm{R}=0.15$ to 0.9 . The concentration ratio varies gradually in the dataset, and it is difficult to separate $\mathrm{He}$ rich and $\mathrm{H}$ rich plasma clearly. Some data is 
intermediate between rich and $\mathrm{H}$ rich. At first, the surveys of the collisionality were performed for the data set including various concentration ratios. Then, effects of the concentration ratio were investigated in low and high collisionality regime..

As shown in Fig.9, $v^{*}{ }_{h}$ dependencies are clearly different in $\chi_{\mathrm{e}}$ and $\chi_{\mathrm{i}} \cdot \chi_{\mathrm{e}}$ increase with decrease of $v^{*}{ }_{h}$ at all radial locations, while $v^{*}{ }_{h}$ dependences of $\chi_{\mathrm{i}}$ are different at different radial locations. At $\rho=0.3 \sim 0.7, v^{*}{ }_{h}$ dependence of $\chi_{i}$ is weak. This is a favourable tendency and suggests $T_{i}$ can increase with higher heating power resulting in lower $v^{*}{ }_{h}$. At $\rho=1.0, \chi_{\mathrm{i}}$ increases with decrease of $v^{*}{ }_{\mathrm{h}}$ as well as $\chi_{\mathrm{e}}$. At $\rho=0.3,0.5, \chi_{\mathrm{i}}$ is lower than $\chi_{e}$, in low $v^{*}{ }_{h}$ regime, while $\chi_{i}$ is higher than $\chi_{e}$ in high $v^{*}{ }_{h}$ regime. Such different tendencies in $\chi_{\mathrm{e}}$ and $\chi_{\mathrm{i}}$ suggest that there is a disconnection between ion and electron transport channels.

Figure 9 indicates that ion transport characteristics are different for low and high collisionality. The normal confinement is in high $v^{*}{ }_{\mathrm{h}}$ regime and ion ITB is in low $v^{*}{ }_{\mathrm{h}}$ regime. It should be noted that the two confinement regimes change gradually and do not show a clear transition like tokamak $\mathrm{L}$ and $\mathrm{H}$ mode.
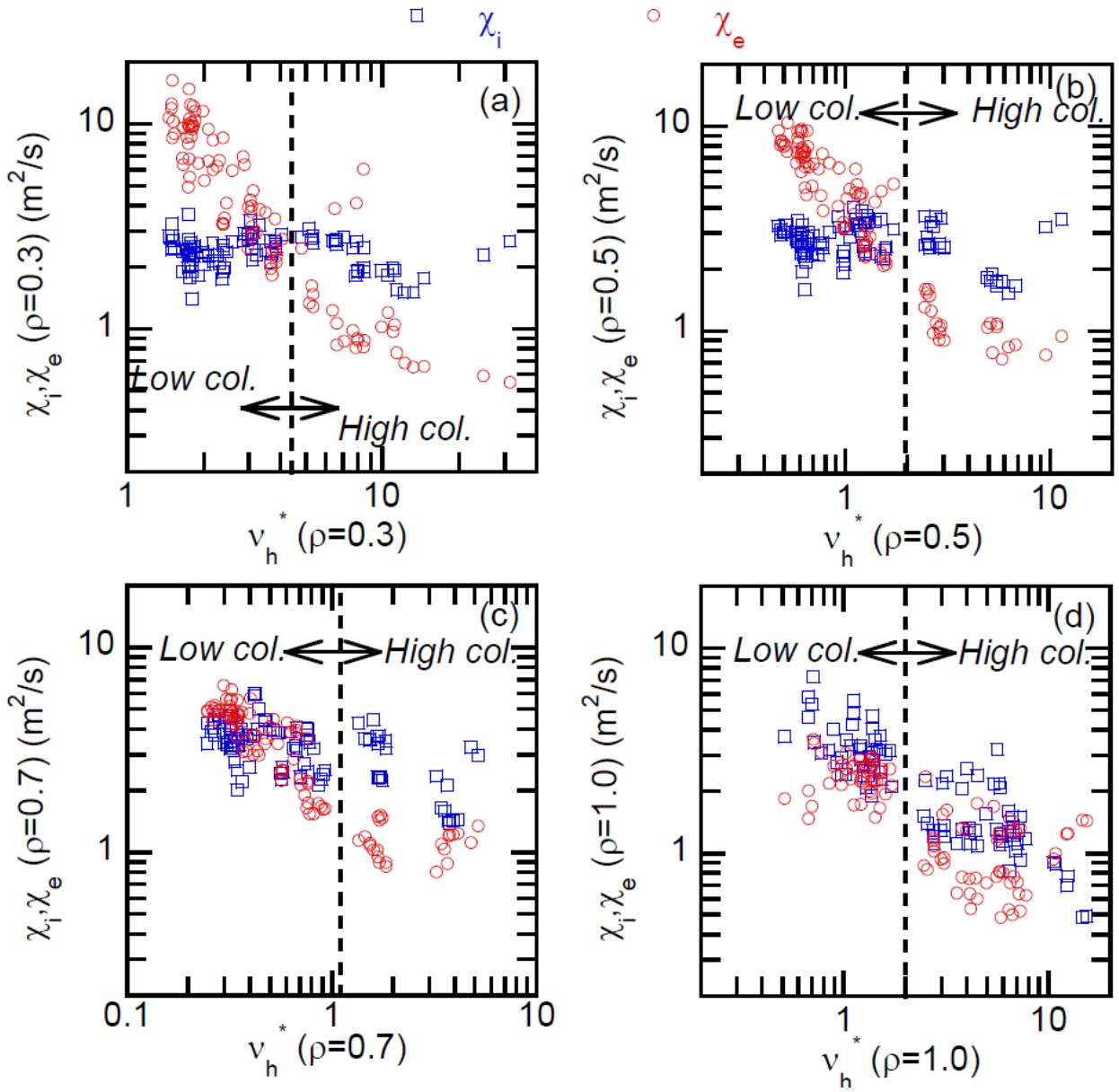
FIG. 9 Collisionality dependence of $\chi_{i}$ and $\chi_{e}$ at different radial location (a) $\rho=0.3$, (b) $\rho=$ 0.5, (c) $\rho=0.7$, (d) $\rho=1.0$.

The dashed lines in (d) indicate the boundary of low and high collisionality regime in Fig.10.

In the following analysis, in order to investigate the ion species effects, low and high collisionality regimes are defined. This is because as described in Section 2-3-2, effects of ion species are different in low collisionality (ion ITB) and in high collisionality (normal confinement).

The dashed lines in Fig. 9 indicate the boundary between low and high collisionality. These are $v_{\mathrm{h}}{ }^{*}=4.4,1.95,1.1$ and 2.0 at $\rho=0.3,0.5,0.7$ and 1.0, respectively. The boundaries approximately correspond to the $v_{\mathrm{h}}{ }^{*}$, where $\chi_{e}$ equals to $\chi_{i}$.

In the dataset, $m_{\text {ieff, }} \mathrm{q}_{\text {ieff }}$ and $\mathrm{T}_{\mathrm{i}}$ vary both in low and high collisionality regime, thus, normalization is necessary and $\chi_{i} / \mathrm{F}_{\mathrm{GB}}$ was used for the survey. The results are shown in Fig. 10. In Fig. 10, low and high $v_{\mathrm{h}}{ }^{*}$ regimes were selected by the boundary which are shown by dashed lines in Fig.9. If ion transport follows gyro-Bohm scaling, the fitted line should be almost flat according to the concentration ratio (horizontal axis). As shown in Fig.10 (a) (c) (at $\rho=0.3-0.7$ ), the data is scattered and it is difficult to argue the concentration ratio dependence. However, in Fig.10 (d), clear tendencies are seen both in low and high collisionality regime at $\rho=1.0$. As shown in Fig.10 (d), $\chi_{i} / \mathrm{F}_{\mathrm{GB}}$ decreases with reduction of the concentration ratio (increase of $\mathrm{He}$ concentration) in low collisionality regime, while it increases in high collisionality regime. These results indicate that ion species is different in low and high collisionality regime and the ion species effect is evident only in plasma edge region. 

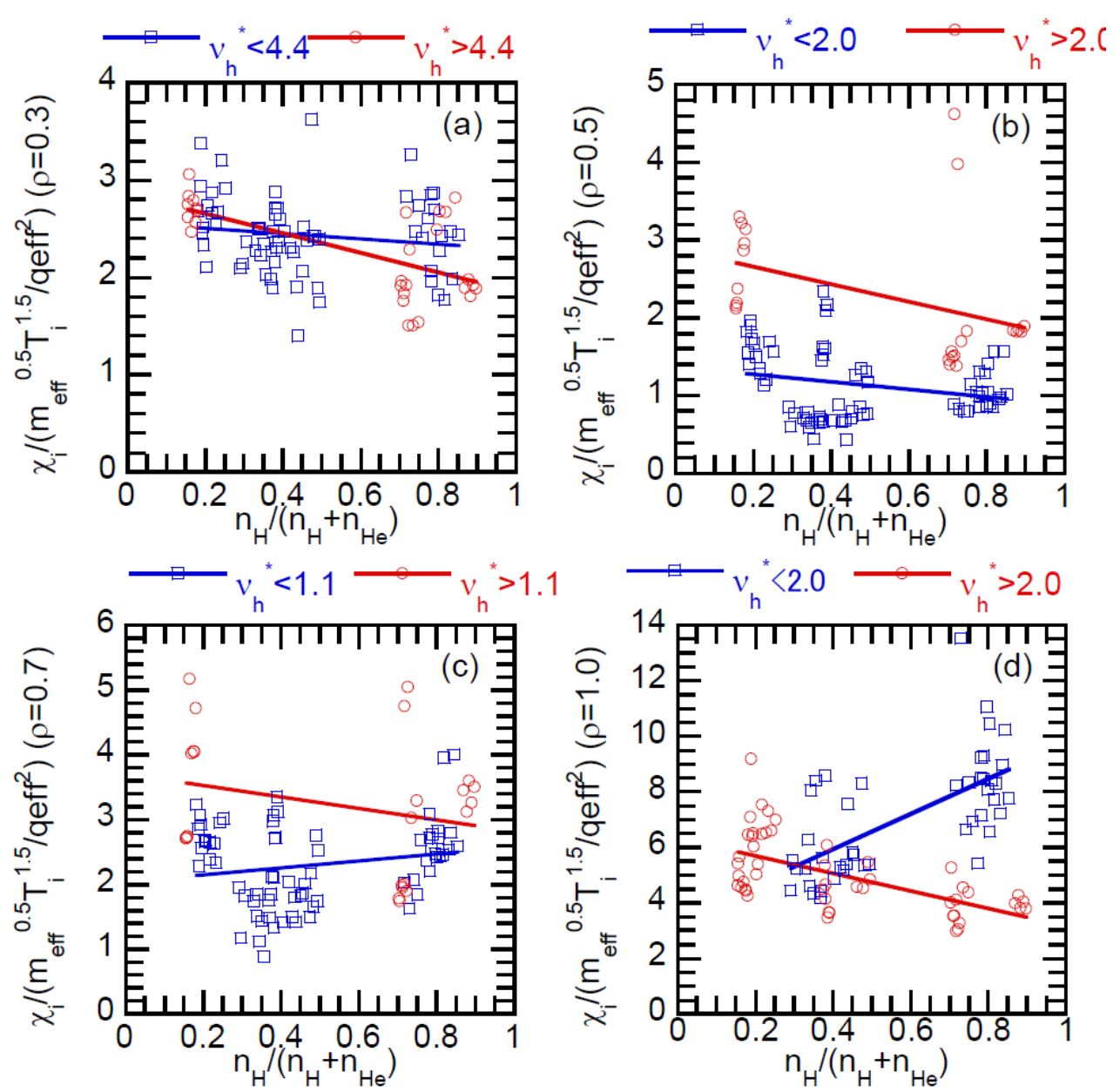

FIG.10 Concentration ratio dependence of normalized $\chi_{i}$ at different radial locations (a) $\rho=$ 0.3, (b) $\rho=0.5$, (c) $\rho=0.7$, (d) $\rho=1.0$.

The data in the low collisionality regime are shown in blue and the data in the high collisionality regime are shown in red.

\section{Turbulence characteristics from the driving term and temporal evolution}

In Section 2, turbulence characteristics are described. In this section, more detail characteristics were investigated from the temporal evolution and driving term.

As described in section 3, clear different spatial profiles were observed in normal and ion ITB plasma both in He rich and $\mathrm{H}$ rich plasma. Such different spatial profile were dynamically visible in the single shot, which changes from normal confinement to ion ITB plasma. Figure 11 shows such an example. In Fig.11, when $\mathrm{T}_{\mathrm{i}}$ and $\mathrm{T}_{\mathrm{e}}$ stays at lower until $1.9 \mathrm{sec}$, turbulence has a peak at $\rho \sim 1.0$. This is the same as well as fluctuation profiles in normal confinement shown in Fig. 2. At $t=1.9 \mathrm{sec}$, additional NBs were injected and both $\mathrm{T}_{\mathrm{e}}$ and $\mathrm{T}_{\mathrm{i}}$ started to increase. At this timing, edge fluctuation (at $\rho \sim 1.0$ ) disappeared, then, core fluctuation $(\rho \sim 0.7)$ started to grow. Both core and edge 
turbulence propagate towards the ion diamagnetic direction in laboratory frame. In Fig.11, core fluctuation $(\rho=0.5-0.9)$ grew after $T_{i}$ increase rather than $T_{e}$ increase. This suggests ion temperature drives the core turbulence.

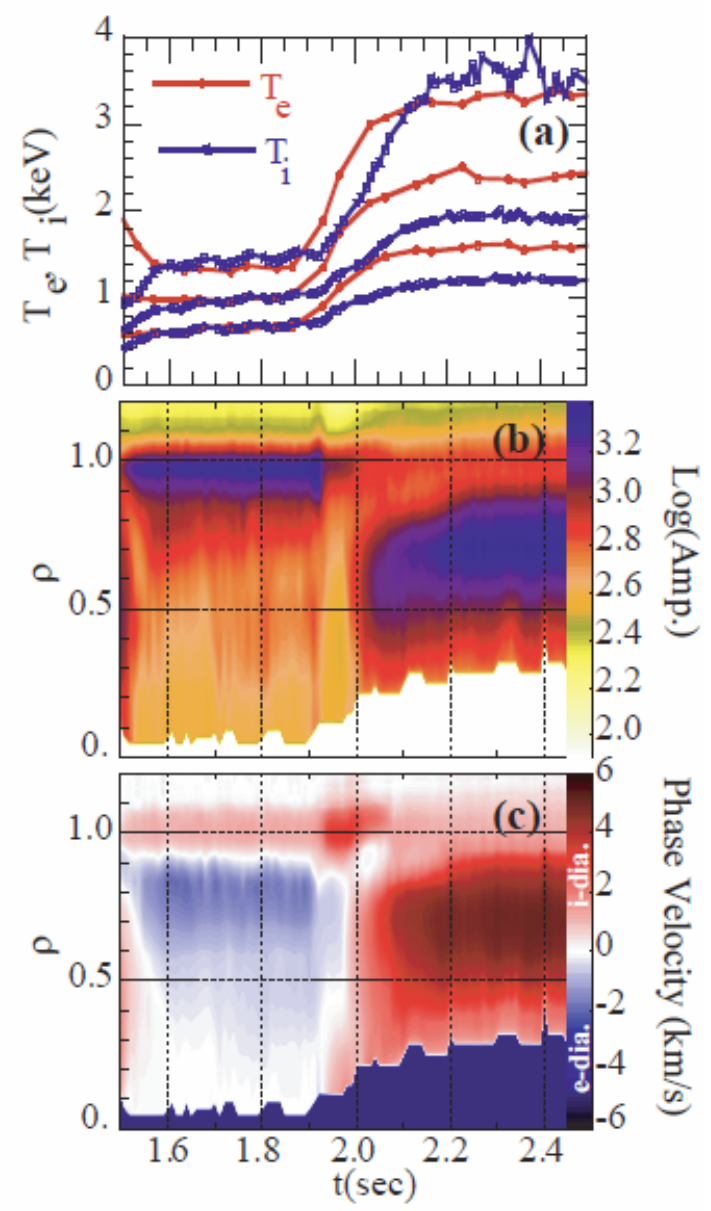

FIG.11 Time history of (a) $T_{e}, T_{i}$, (b) fluctuation amplitude and (c) fluctuation phase velocity in the laboratory frame

In Fig. $11(a)$, the radial locations $T_{e}$ and $T_{i}$ are $\rho=0.3,0.7$ and 0.9 from the upper trace. The white and dark blue regions in Fig.11 (b) and (c) each indicate inaccessible region of the measurements. Ion species is hydrogen [50].

The driving term of turbulence was investigated from the same data set as transport analyses were performed in Section 3. Since electron density varies in the data set, fluctuation level, which is defined as the fluctuation amplitude normalized by the electron density, was used for the investigations. Figure 12 shows parameter dependence of core turbulence at $\rho=0.7$. The position $\rho=0.7$ is the peak position of turbulence in ion ITB plasma. Data include both $\mathrm{He}$ and $\mathrm{H}$ rich plasmas. Fluctuation levels from upper and 
lower sides are shown by different symbols. However, the dependences are rather similar. As shown in Fig. 12 (a), the fluctuation level increases with increase of $\mathrm{L}_{\mathrm{Ti}}{ }^{-1}$, while there is not a clear dependence on $\mathrm{L}_{\mathrm{Te}}{ }^{-1}$, and $\mathrm{L}_{n e}{ }^{-1}$ as shown in Fig.12 (b) and (c). This is consistent with the result of linear calculation of core turbulence, which shows that the most dominant instability is ITG. This is in clear contrast to the turbulence characteristics of ECRH plasma of ATF [51,52] and ECRH+NBI plasma of Heliotron-E [53]. The core fluctuation in both device increases with increase of electron density gradient. In ATF, turbulence was identified to be dissipative trapped electron mode, in Heliotron-E to be collisionless circulating electron drift wave.

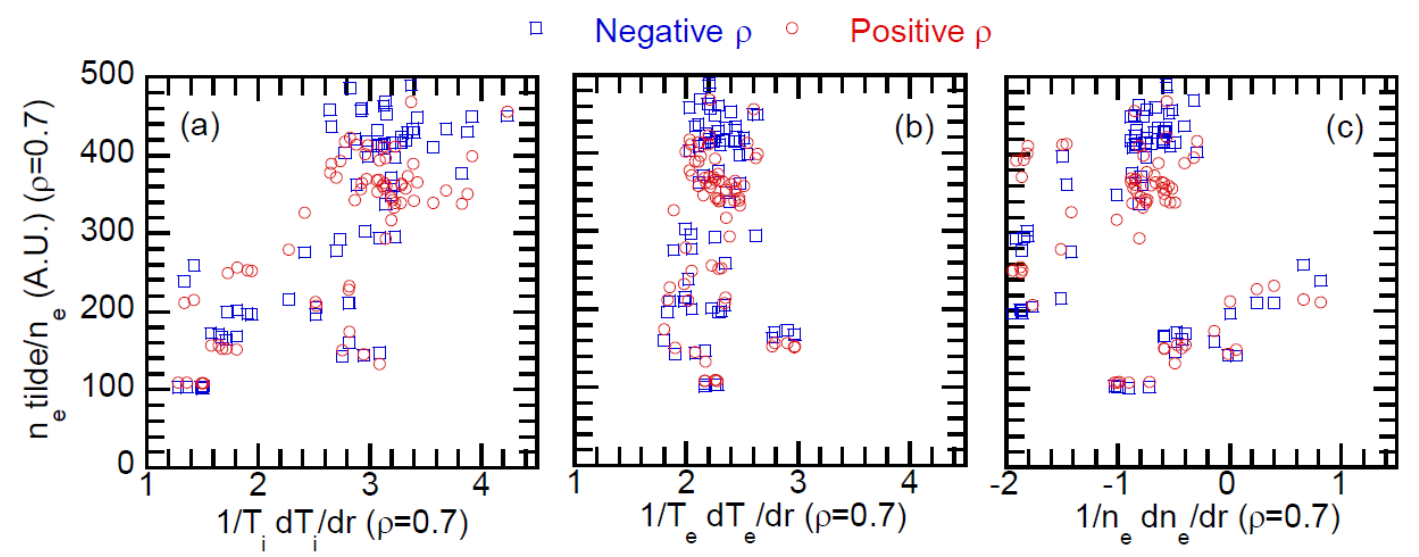

FIG.12 Parameter dependence of fluctuation saturation level at $\rho=0.7$ on (a) $L_{T i}{ }^{-1}$, (b) $L_{T e}{ }^{-1}$ and (c) $L_{n e}{ }^{-1}$. Red circles and blue squares indicate positive and negative r respectively.

The edge fluctuation ( $\rho \sim 1.0$ ) did not show clear dependence with $\mathrm{L}_{\mathrm{Ti}^{-1}}, \mathrm{~L}_{\mathrm{Te}}{ }^{-1}$ and $\mathrm{L}_{\mathrm{ne}}{ }^{-}$ ${ }^{1}$. An interesting observation is concentration ratio dependence of edge fluctuation of normal confinement. As shown in Fig. 6 (a-2), fluctuation amplitude is clearly smaller in He rich than in $\mathrm{H}$ rich plasma. The systematic survey is shown in Fig. 13. The edge fluctuation level in high collisionality regime decreases with increase of He concentration. More detail investigation is necessary to identify edge fluctuation. 


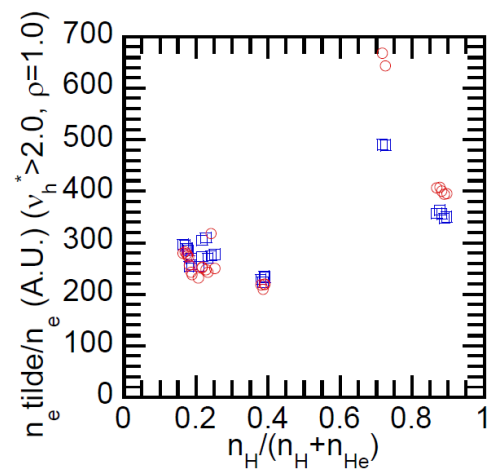

FIG. 13 Edge fluctuation level dependence on concentration ratio in high collisionality regime

There is a clear difference of turbulence characteristics in normal confinement and ion ITB plasma. As shown in Fig.6, in normal confinement, fluctuation amplitude are smaller in He rich plasma but in ion ITB plasma, the time averaged fluctuation profiles are almost identical. However, fast time response analysis in ion ITB plasma showed clear difference of the turbulence response. Figure 14 shows fast time response of RF probe signal, fluctuation phase velocity and fluctuation power at $\rho=0.7$. The position of $\rho=0.7$ is the peak position of ITB plasmas. RF probe measures $400 \mathrm{MHz}$ ion cyclotron emissions, which can show fast ion loss qualitatively [54]. As shown in Fig.14, when the spike appeared in RF probe, change of the phase velocity and clear reduction of the decrease of the ion diamagnetic fluctuation and small increase of electron diamagnetic fluctuation are observed. This observation is the same as the observation of fast ion driven resistive interchange mode $[55,56]$. When the fast ion is lost, strong negative $E_{r}$ and $E_{r}$ shear can be formed $[55,56]$. As shown in Fig.14 (a-2), (b-2), change of the phase velocity at each burst is $5-10 \mathrm{~km} / \mathrm{s}$. This corresponds approximately $25-50 \mathrm{kHz} \mathrm{E}_{\mathrm{r}} \mathrm{xB}_{\mathrm{t}}$ shearing rate assuming phase velocity is dominated by $\mathrm{E}_{\mathrm{r}} \mathrm{xB}_{\mathrm{t}}$ rotation velocity. The estimated shearing rate is much higher than the growth rate as shown in Fig. 7(a). The suppression of the turbulence in Fig.14 (a-3) and (b-3) is likely to be due to the stabilization of $\mathrm{E}_{\mathrm{r}} \mathrm{xB}_{\mathrm{t}}$ shear induced by the fast ion loss. An important observation is the frequency of the bursting signal. A burst appears every $20 \mathrm{msec}$ in He rich plasma, and every $3 \mathrm{msec}$ in H-rich plasma. This more frequent burst in $\mathrm{H}$-rich plasma shows larger amplitude modulation in H-rich plasma.

The burst and transient reduction of the turbulence appears at a much higher frequency in H-rich plasma, although time averaged fluctuation amplitude are almost the same. This suggests that fluctuation between bursts is higher in H-rich plasma. As shown in Fig.14 (a-3) and (b-3), fluctuation power in stationary phase in He-rich plasma is lower than those in inter-burst in H-rich plasma. For example the averaged power of He-rich at $\mathrm{t}=$ 
4.7092-4.7197 sec, which is the region shown by green dashed line in Fig.14 (a-3) is 0.61. On the other hand, the averaged power of H-rich at $\mathrm{t}=4.7113-4.736 \mathrm{sec}$, which is the region shown by green dashed line in Fig.13 (b-3) is 0.86 . The former is $71 \%$ of latter one. Such difference of fluctuation power between stationary phase and inter-burst may affect the core confinement property.

The different characteristics of the fast ion driven instability may affect bulk ion transport, as well. If loss of ion heating power is significant in H-rich plasma, the difference of the ion confinement between He rich and H-rich plasma becomes smaller, since power deposition does not include the loss due to the fast ion driven instability. However, the preliminary analysis showed that each burst loses are around $1 \mathrm{~kJ}$ of energy, while net stored energy is about1MJ. It is likely that loss of the fast ion does not affects transport analyses reported in this paper. More detail analysis comparing other fast ion diagnostics such as neutral particle analyzer is now under way and being prepared for the publications [57]. The bursting signals were not observed both in $\mathrm{H}$ and $\mathrm{He}$ rich plasma of normal confinement of high $v_{\mathrm{h}}{ }^{*}$ regime.
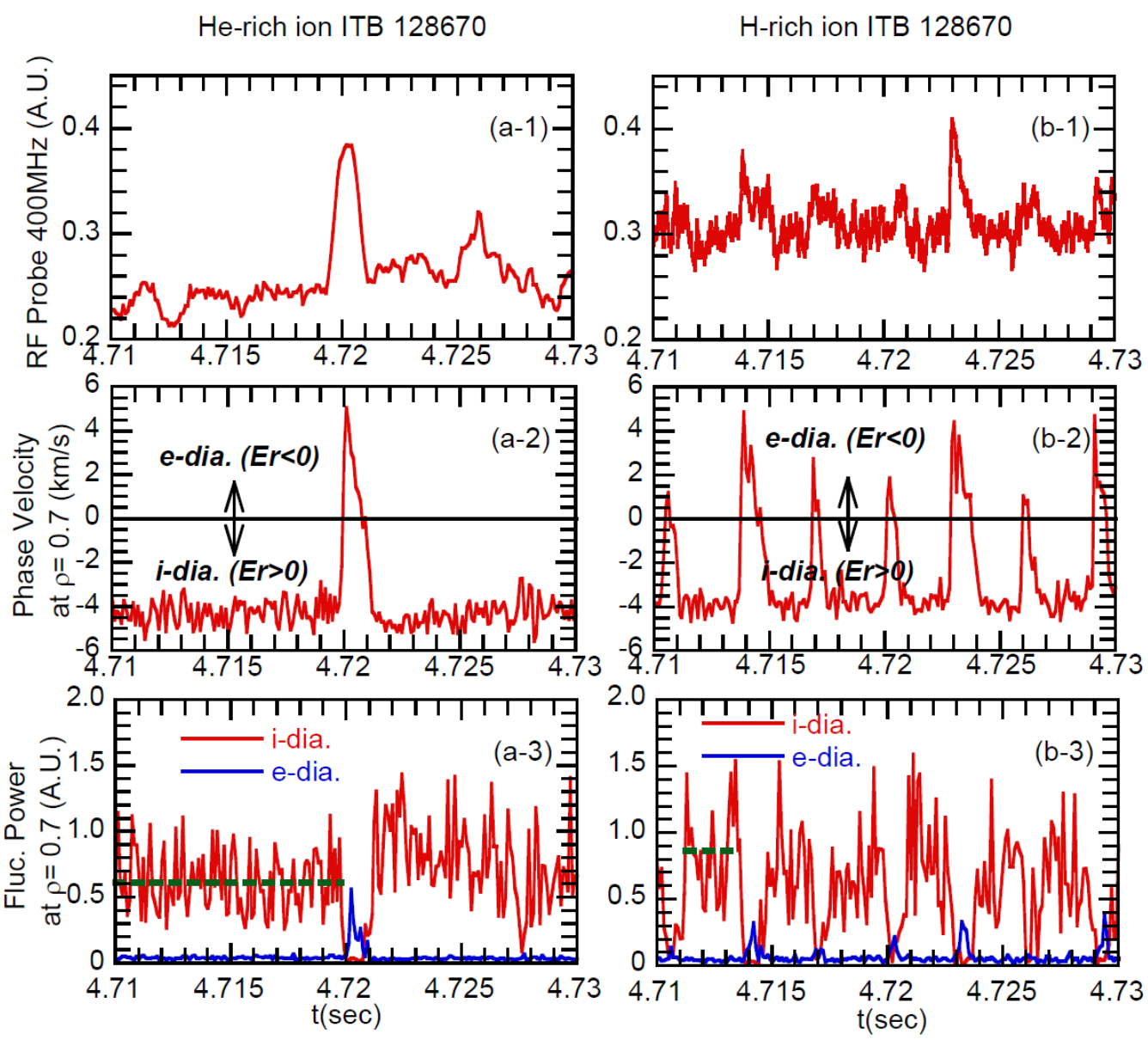
Fig.14 Temporal history of (a-1),(b-1), (a-2),(b-2) RF probe, fluctuation phase velocity at $\rho=0.7$ and (a-3),(b-3) fluctuation power at $\rho=0.7$

(a-1) (a-3) in He rich, (b-1) (b-3) in H-rich of ITB plasma. Green dashed lines indicate averaged fluctuation power.

\section{Discussion and summary}

Systematic and detailed surveys of heat transport were performed in LHD scanning collisionality and $\mathrm{H} / \mathrm{He}$ concentration ratio. Electron and ion energy transports were characterized by normal confinement (low power heating and high density) and ion ITB plasma (high power heating and low density). The change of two confinement statuses are gradual. Experimental $\chi_{\mathrm{e}}$ increase with decrease of collisionality at almost entire region of plasma $(\rho=0.3 \sim 1.0)$. On the other hand, experimental $\chi_{i}$ almost stays constant at $\rho=0.3 \sim 0.7$ and increases with decrease of collisionality at $\rho=1.0$. At higher collisionality with lower heating power, $\chi_{\mathrm{i}}$ is higher than $\chi_{\mathrm{e}}$. This is characteristic of normal confinement. At lower collisionality with higher ion heating power, $\chi_{i}$ is smaller than $\chi_{\text {e. at }} \rho=0.3$ and 0.5 . This is characteristic of ion ITB plasma.

Modest increase of $\mathrm{T}_{\mathrm{i}}$ was observed with increase of He concentration ratio in normal confinement plasma. This is due to the increase of deposition power per ion. In normal confinement, the entire region was ion root. Experimental $\chi_{i}$ is a factor $1.2 \sim 2$ higher than neoclassical $\chi_{i}$ at $\rho<0.8$ and the difference becomes smaller at $\rho>0.8$. Experimental $\chi_{\mathrm{e}}$ is comparable with neoclassical $\chi_{\mathrm{e}}$. at $\rho<0.8$. Deviation of experimental $\chi_{\mathrm{e}}$ from neoclassical $\chi_{\mathrm{e}}$ becomes higher at $\rho>0.8$. The contribution of neoclassical transport is larger than the previous results of $\mathrm{H}$ rich normal confinement plasma [16]. The larger neoclassical contribution of present results are due to smaller absolute $\mathrm{E}_{\mathrm{r}}$ compared with previous results.

Clear increase of $T_{i}$ was observed in He rich plasma of ion ITB. This is due to the reduced transport. In ion ITB plasma, electron root is found at $\rho>0.75$. In this region, neoclassical contribution becomes smaller both in $\chi_{\mathrm{i}}$ and $\chi_{\mathrm{e}}$. On the other hand, at $\rho<$ 0.75 , GSRAKE outputs ion root at $\rho<0.75$. However, it is likely that co-dominant toroidal torque can induce electron root with positive $E_{r}$ and reduces neoclassical transport. The positive $E_{r}$ at $\rho<0.75$ is partly supported by the ion diamagnetic propagation of the fluctuation at $\rho=0.4 \sim 1.0$. At $\rho<0.75$, the estimated neoclassical $\chi_{i}$ and $\chi_{\mathrm{e}}$ are likely an overestimation, and the anomalous contribution will be larger than the present estimation. These characteristics of neoclassical transport are not very different in $\mathrm{He}$ and $\mathrm{H}$ plasma. More precise estimations of neoclassical transport taking into account effects of multi ion species and external torque input are necessary. 
Both in normal and ion ITB plasma, edge $T_{i}$ pedestal was formed. In normal confinement, $\mathrm{L}_{\mathrm{Ti}}{ }^{-1}$ of core region, which is the inside of edge pedestal, is smaller in He rich than in $\mathrm{H}$ rich plasma. This results in comparable $\mathrm{T}_{\mathrm{i}}$ in the central region. In ion ITB, $\mathrm{L}_{\mathrm{Ti}^{-1}}{ }^{-1}$ of core region are almost identical. Then, higher edge pedestal $\mathrm{T}_{\mathrm{i}}$ in He rich plasma introduces higher central $\mathrm{T}_{\mathrm{i}}$ due to the profile stiffness. The $\mathrm{L}_{\mathrm{Ti}}{ }^{-1}$ of core region are likely determined by ITG threshold.

In order to investigate the confinement improvement of ion transport due to the different $\mathrm{He}$ and $\mathrm{H}$ concentration ratio, normalization by gyro-Bohm factor were performed. The gyro-Bohm factor includes the effects of effective mass and effective charge. After normalization, improvements are only visible in the plasma edge region of ion ITB plasma. This likely induce higher edge pedestal $T_{i}$.

Presently, quantitative argument of anomalous contribution is difficult. Non linear study of $\mathrm{H}$ rich ion ITB plasma showed that anomalous ion and electron heat flux is well accounted by the ITG driven flux [55-58]. Electron temperature gradients (ETG) mode are also linearly unstable [57]. However, ETG does not contribute the electron heat flux [57]. Quasi linear study of shots in Fig. 4 were reported in ref. 45. Non linear studies are now underway.

The difference of transport characteristics are associated with different turbulence characteristics. In normal confinement, fluctuation peak is at $\rho=1.0$. The edge fluctuation disappears when ion ITB formed and $T_{i}$ increase. The core fluctuation peak at $\rho=0.7$. Core fluctuation is likely to be ITG.

In normal confinement, fluctuation level is smaller in He rich plasma than in $\mathrm{H}$ rich plasma. In ion ITB, fluctuation level is almost identical in $\mathrm{He}$ and $\mathrm{H}$ rich plasma. However, a clear difference of the fast time response was found. In $\mathrm{H}$ rich, turbulence is suppressed transiently when fast ions were lost. This is likely to be formation of strong $E_{r}$ shear due to the fast ion loss. Such suppression was much less frequent in He rich plasma, and higher fluctuation power between the bursts was found in $\mathrm{H}$ rich plasma. The higher fluctuation power between the burst in $\mathrm{H}$ rich plasma may enhance transport.

In this paper, only the energy transport was studied. The next step is to investigate particle and impurity transport. Particle transport was briefly investigated from the averaged electron density and particle source. The particle source is the sum of $\mathrm{H} \alpha$ and twice of HeI line. H $\alpha$ and HeI lines are cbsolutely calibrated. The ratio $\mathrm{n}_{\mathrm{e} \_ \text {bar }} /(\mathrm{H} \alpha+2 \mathrm{HeI})$ gives global particle confinement time in arbitrary unit. The global particle confinement time showed clear dependence on concentration ration as shown in Fig.15. However, the particle confinement time did not show clear dependence on $v_{\mathrm{h}}{ }^{*}$. Both in normal confinement and ion ITB plasma, particle confinement time is shorter in He rich than in 
$\mathrm{H}$ rich plasma. This is favorable for $\mathrm{He}$ ash exhausting. For the further investigation, estimation of diffusion coefficient and convection velocities and their parameter dependences are necessary. This is possible by density modulation experiments, which have been already performed for hydrogen normal confinement [13]

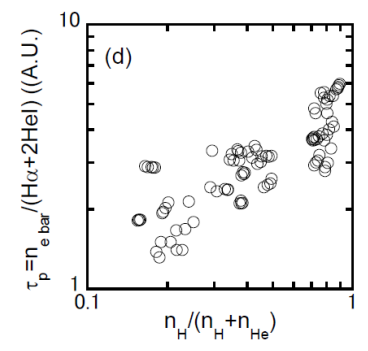

Fig. 15 Concentration ratio dependence of global particle confinement time.

The survey performed in this paper is still in the limited experimental regime of LHD. For example, further systematic survey for higher $T_{e} / T_{i}$ and lower $v_{h}{ }^{*}$ using ECRH, for higher $n_{\mathrm{e}}$ for higher $v_{\mathrm{h}}{ }^{*}$ using pellet injection and strong gas puffing and for high $\beta$ and different $\rho^{*}$ with lower $\mathrm{B}_{\mathrm{t}}$ should be carried out in order to provide the complete picture of transport in LHD. Such studies are necessary for the heliotron-type reactor design. Also, the results obtained here will be good reference material for investigating the hydrogen isotope effects. The deuterium experiment of LHD, which is starting in 2017, will provide an important database to understand effects of ion mass and charge number on transport.

\section{Acknowledgments}

This work is supported by NIFS grants NIFSULHH013, NIFS10ULRR702, NIFSULHH004, ULHH005, ULHH02 ULHH028, NIFS16KLER045 and NIFS14UNTT006

\section{References}

[1] IIYOSHI, A. et al, Nucl. Fusion 3 (1999), 1245

[2] MASUZAKI, S. et al, Fusion Eng. Des. 85, (2010), 940

[3] Special issue on Large Helical Device, Fusion Sci. Tech. 58, (2010), 1

[4] SAGARA, A. et al, Fusion Eng. Des. 89, (2014) 2114

[5] MURAKAMI, $M$ et al, 'Dimensionless scaling of confinement in ATF ', presented at $13^{\text {th }}$ IAEA Intl. Conf. on Fusion Energy, Washington, DC, 1990

[6] SUDO, S. et al, Nucl. Fusion 30, (1990), 11

[7] STROTH, U. et al, Nucl. Fusion 36, (1996), 1063

[8] YAMADA, H. et al, Fusion Sci. Tech. 46, (2004), 82 
[9] YAMADA, H. et al, Nucl. Fusion, 45, (2005), 1684.

[10] ITER Physics Basis Nucl. Fusion 39, ( 1999), 2178

[11] FUNABA, H. et al, Plasma Fusion Res., 3, (2008),022

[12] WATANABE, K.Y, et al, Physics of Plasmas, 18, (2011), 056119

[13] TANAKA, K. et al, Fusion Sci. Tech. 58, (2010), 70

[14] SUDO, S. et al, Plasma Phys. Control. Fusion, 58, (2016), 043001

[15] YOKOYAMA, M. et al, Nucl. Fusion, 47, (2007), 1213

[16] DINKLAGE, A. et al, Nucl. Fusion, 53, (2013), 063022

[17] STROTH, U. et al, Phys. Scr., 51, (1995), 655

[18] TANAKA, K. et al, Plasma Phys. Control. Fusion, 58, (2016), 055011

[19] OTANI, Y. et al, "Initial Result of Density Modulation Experiment for Study of Hydrogen Isotope Effects on Particle Transport in Heliotron J", to be submitted, J. Phys. Soc. Jpn.

[20] ZURO, B. et al, Plasma Phys. Control. Fusion, 56, (2014), 124007

[21] ASDEX Team, Nucl. Fusion 29, (1989), 1959

[22] RYTER, F. and H-Mode Database Working Group, Nucl. Fusion 36, (1996), 1217

[23] LACKNER, K. et al, Plasma Phys. Control. Fusion 36, (1994), B79

[24] RIGHI, E. et al, Nucl. Fusion 39, (1999), 309

[25] URANO, H. et al, Phys. Rev. Lett., 109, (2012), 125001

[26] RYTER, F. et al, Nucl. Fusion, 49, (2009), 062003

[27] BESSERODT-WEBERPALS, M. et al, Journal of Nuclear Materials 196-198 (1992) 943

[28] MAKINO, R. et al, "Heat and particle transport in hydrogen and helium ECH plasmas on LHD”, presented at $41^{\text {st }}$ European Physical Society Conference on Plasma Physics, Berlin, 2014, P4.087

[29] NAGAOKA, K. et al, Plasma Fusion Res. 11, (2016), 2402106

[30] IDA, K. et al, Phys. Plasma 16, (2009), 056111

[31] YAMADA, I. et al, Fusion Sci. Tech. 58, (2010) 345

[32] AKIYAMA, T. et al, Fusion Sci. Tech. 58, (2010) 352

[33] TANAKA, K. et al, Plasma Fusion Res 3, (2008) 050

[34] YOSHINUMA, M. et al, Fusion Sci. Tech. 58, (2010) 37

[35] TANAKA, K. et al, Rev. Sci. Instrum. 79, (2008), 10 E702

[36] MICHAEL, C. et al, Rev. Sci. Instrum. 86, (2015), 093503

[37] YOKOYAMA, M. et al, "Extended Capability of the Integrated Transport Analysis Suite, TASK3D-a, for LHD Experiment, and its Impacts on 
Facilitating Stellarator-Heliotron Research"[FIP/P7-35], paper presented at $25^{\text {th }}$ IAEA Int. Conf. on Fusion Energy, Saint Petersburg, 2014

[38] BEIDLER, C.D. and D'HAESELEER, W.D. Plasma Phys. Control. Fusion 37, (1995),463

[39] SPONG, D.A. et al, Nucl. Fusion, 47 (2007), 626

[40] MICHAEL, C. et al, J. Plasma Fusion Res. 2, (2007), S1034

[41] MIKKELSEN, D. R. Phys. Plasmas 21, (2014), 082302

[42] TAKAHASHI, H. et al, ' Extension of Operational Regime in HighTemperature Plasmas and Effect of ECRH on Ion Thermal Transport in the LHD', submitted to Nucl. Fusion

[43] DORLAND, W., et al, Phys. Rev. Lett. 85, (2000), 5579

[44] MIKKELSEN, D. R. Phys. Plasmas 21, (2014), 112305

[45] PUSZTAI, I., CANDY, J., and GOHIL, P., Phys. Plasmas 18, (2011)

[46] BUSTOS, A. Phys. Plasmas 22, (2015) 012305

[47] NUNAMI, M. et al, Phys. Ctrl. Fusion 59, (2017) 044013

[48] MURAKAMI, S. Nucl. Fusion 42, (2002) L19-L2

[49] YOKOYAMA, M. et al, J. Plasma Fusion Res. 81, (2005) 83

[50] TANAKA, K. et al, Plasma Fusion Res. 5, (2010), S2053

[51] WILGEN, J, B. et al, Phys. Fluids B 5(7),(1993),2513

[52] SHATS, M. G. et al, Phys. Plasma 2 (1995), 398

[53] KADO, S. et al, J. Phys. Soc. Jpn 65 (1996), 3434

[54] THATIPAMULA, S. G., et al, Plasma Phys. Control. Fusion, 58, (2016), 065003

[55] DU, X. D. et al, Phys. Rev. Lett. 114, (2015), 155003

[56] DU, X. D. et al, Nucl. Fusion, 56, (2016), 016002NUNAMI, M. et al, Plasma Fusion Res. 6, (2011), 1403001

[57] MICHAEL, C. private communication

[58] NUNAMI, M. et al, Phys. Plasmas 19, (2012), 042504

[59] ISHIZAWA, A. et al, Nucl. Fusion 55 (2015) 043024

[60] ISHIZAWA, A. et al, Nucl. Fusion 57 (2017) 066010 